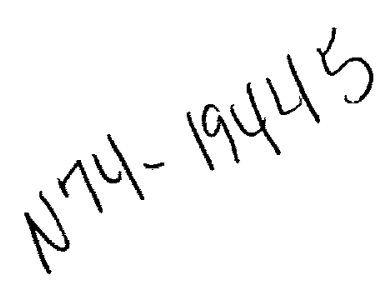

\title{
9. Spectral Analysis of Four Meteors
}

\author{
Gale A. Harvey \\ Langley Research Center, NASA \\ Hampton, Virginia
}

\begin{abstract}
Four meteor spectra from the NASA LRC Faint Meteor Spectra Patrol are analyzed for chemical composition and radiative processes. The chemical compositions of the Taurid, Geminid, and Perseid meteors were found to be similar to that of a typical stony metearite. The chemical composition of the sporadic meteor was found to be similar to that of a nickel-iron meteorite. The radiation from optical meteors $(+1$ to -10 absolute photographic magnitude) was found to be similar to that of a low-temperature gas, except that strong, anomalous ionic radiation is superposed on the neutral radiation in bright $(<-3$ mag), fast meteors.
\end{abstract}

$\mathrm{D}$ ERING THE 1960s, THE ACTIVITY IN SPACE resulted in a strong effort dirceted toward determining the meteoroid environment and its attendant hazard to spacecraft. By 1971, lack of definitive meteoroid damage to spacecraft had shown that the metcoroid environment did not present a significant danger to most missions. At the same time, the measured uncertainties in the mass flux of meteoroids, over a wide range of mass, had been reduced from orders of magnitude to factors of 3 to 10 (Ayres et al., 1970; Cook et al., 1963; D'Aiutolo et al., 1967; Harvey, 1970). However, the multitude of observations (Grygar et al., 1968; Harvey, 1971a; Lindblad, 1963; Millman, 1967) during this time period clcarly indicated that meteoroids were very heterogencous in nature and that the simplificd concept of a mass-flux representation of the metcoroid environment had limitations. By 1970, the statistieal or gross meteoroid environment was fairly well determined. However, many of the techniques that are applied to the study of the overall environment are not well suited to a refined study of individual metcoroids.

Meteor spectra research being conducted by NASA Langley Researeh Center is intended as a detailed study of individual metcoroids. This rescarch is based on the techniques and principles of quantitative spectroscopy. The data for this research are obtained from the NASA IRC Faint Meteor Spectra Patrol (Harvey, 1971a). This patrol provides statistical quantities (several hundred per year) of meteor spectra. These spectra are used for detailed measurements of composition, for statistical studies of composition, and for study of meteor radiation.

This paper presents the results of the detailed analysis of four of the better ( $>100$ lines) meteor spectra obtained from the patrol. The approaeh used in these analyses is to measure the population of excited states of the motcoric gas, and to use the parameter obtained from these measurements to obtain quantitative elemental ehemical composition of the initial meteoroid. Meteor radiation processes are discussed. The "effective radiation temperatures" and "derived meteoroid compositions" are presented. Prior to this present effort only three meteor spectra are known to have been quantitatively analyzed for composition (Ceplecha, 1964, 1965; Harvey, 1970).

The NASA LRC Faint Meteor Spectra Patrol (Harvey, 1971:a) has been obtaining metcor 
spectra since November 1968. The patrol uses speciall, designed Maksutov slitless spectrographs and a photoelectric meteor-detectionshutter system. By the end of 1970, approximately 500 meteor spectra had been obtained by the patrol. Four of these were selected as useful for detailed spectroscopic analysis and as gencrally representative of the brighter meteors that had been photographed. Further, the meteoroids that produced these four spectra are also generally representative of the metcor velocity range. One of the spectra is of a slow sporadic meteor. The other three spectra are of Taurid, Geminid, and Perseid meteors, respectively.

\section{SPORADIC METEOR}

The spectrum of the slow, sporadic meteor is shown in figure 1. This spectrum was recorded during the night of April 4, 1969, and no position or orbital data are available. A velocity of 20 $\mathrm{km} / \mathrm{s}$ or less is estimated for the meteor on the basis of a lower "effective radiation temperature" measurement than that obtained for the $28 \mathrm{~km} / \mathrm{s}$ Taurid. The meteor spectrum was photographed with a $150-\mathrm{mm}$ aperture, $f / 1.3$ Maksutov slitless spectrograph equipped with a 407 line $/ \mathrm{mm}$ diffraction grating. Typical slow-meteor values of $90 \mathrm{~km}$ initial height, trajectory-optical axis angle of $45^{\circ}$, and a duration of $2 \mathrm{~s}$, were used to com- pute the intensity of this meteor. By comparing the computed intensity of this meteor in the blue and near ultraviolet region with that of an artificial meteor (Harvey, 1967a), an absolute photographic magnitude brighter than -10 is obtained for the meteor. As can be seen in figure 1 , this bright meteor was recorded in the third, fourth, and fifth orders of the spectrum with dispersions of 40,32 , and $24 \AA / \mathrm{mm}$, respectively. One hundred and twenty-two of the strongest lines at point $A$ in the spectrum have been identified in a preliminary wavelength analysis and are listed in table 1. The first column of table 1 lists the wavelengths measured from the microdensitometer tracing, column two lists the identified wavelengths (Corliss and Bozman, 1962; Moore, 1945), column three lists the multiplet numbers from Moore (1945), column four lists the statistical weight-Einstein transition probability products from Corliss and Bozman (1962). Columns five and six list the upper and lower energy levels of the atomic transitions as listed in Moore (1945). Most of the lines are from ground state multiplets of iron. The large number of lines and the good spectral resolution lend themselves to a detailed analysis. The spectrum was recorded on a blue sensitive emulsion and covers the wavelength interval of $3100 \AA$ to $4600 \AA$ in partially overlapping orders.

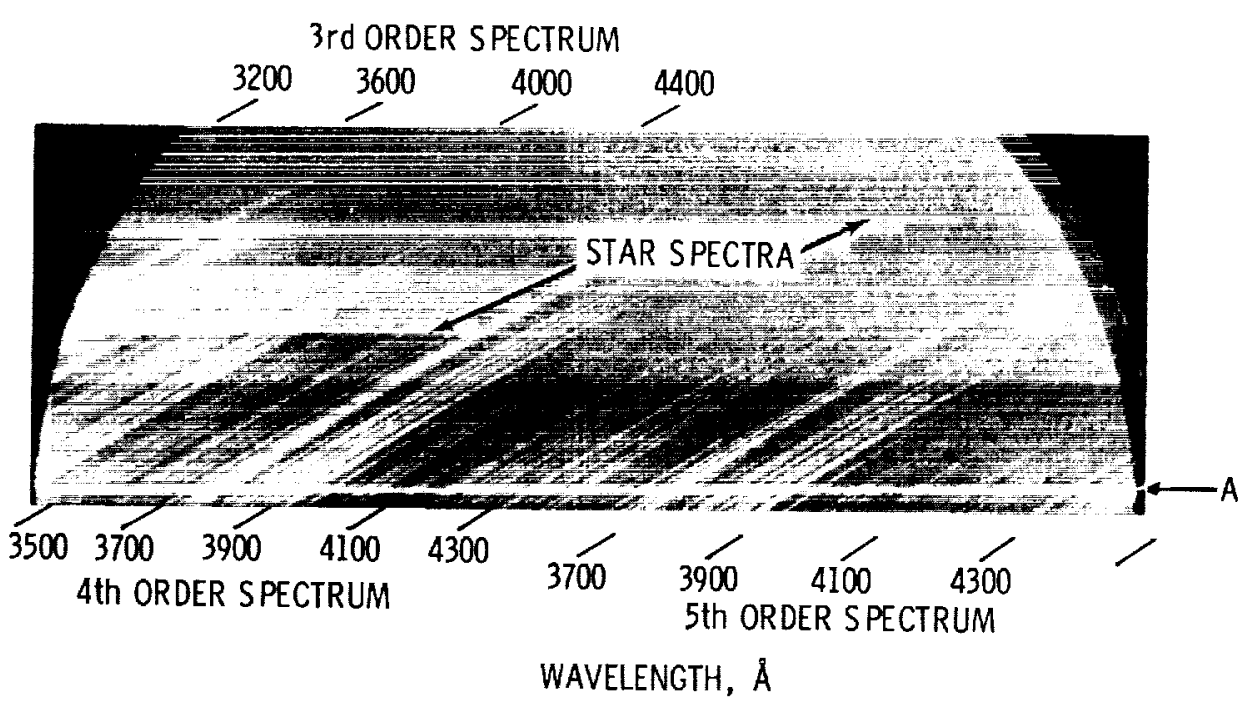

FIGURE 1.-Enlargement of spectrogram of a sporadic meteor. 
SPECTRAL ANALYSIS OF FOUR METEORS

TABLE 1.-Wavelength Identifications of Sporadic Meteor

\begin{tabular}{|c|c|c|c|c|c|}
\hline $\begin{array}{c}\lambda \text { measured } \\
(\AA)\end{array}$ & $\begin{array}{c}\lambda \text { identified } \\
(\AA)\end{array}$ & Multiplet no. & $\begin{array}{c}g A \\
\left(\times 10^{8} / \mathrm{s}\right)\end{array}$ & $\begin{array}{c}E_{1} \\
(\mathrm{eV})\end{array}$ & $\begin{array}{c}E_{2} \\
(\mathrm{eV})\end{array}$ \\
\hline 3424.8 & 3424.29 & $81 \mathrm{Fe}$ & 17 & 2.17 & 5.77 \\
\hline 3426.5 & 3426.39 & $82 \mathrm{Fe}$ & 7.4 & 2.17 & 5.77 \\
\hline & 3426.64 & $82 \mathrm{Fe}$ & 7.7 & 2.19 & 5.79 \\
\hline & 3427.12 & $81 \mathrm{Fo}$ & 34 & 2.17 & 5.77 \\
\hline 3429.0 & 3428.20 & $81 \mathrm{Fc}$ & 8.8 & 2.19 & 5.79 \\
\hline 3433.0 & 3433.04 & $23 \mathrm{Co}$ & 9.1 & 0.63 & 4.22 \\
\hline & 3433.60 & $52 \mathrm{Cr}$ & 44 & 2.53 & 6.13 \\
\hline 3436.5 & 3436.19 & $52 \mathrm{Cr}$ & 26 & 2.53 & 6.13 \\
\hline 3440.6 & 3440.61 & $6 \mathrm{Fe}$ & 2.8 & 0.00 & 3.59 \\
\hline & 3440.99 & $6 \mathrm{Fe}$ & 0.64 & 0.05 & 3.64 \\
\hline 3444.0 & 3443.88 & $6 \mathrm{Fe}$ & 0.34 & 0.09 & 3.67 \\
\hline 3446.0 & 3445.15 & $81 \mathrm{Fe}$ & 17 & 2.19 & 5.77 \\
\hline & 3446.26 & $20 \mathrm{Ni}$ & 3.8 & 0.11 & 3.69 \\
\hline 3447.5 & 3447.28 & $82 \mathrm{Fe}$ & 5.3 & 2.19 & 5.77 \\
\hline 3450.5 & 3450.33 & $82 \mathrm{Fe}$ & 8.9 & 2.21 & 5.79 \\
\hline 3452.5 & 3452.28 & $25 \mathrm{Fe}$ & 0.49 & 0.95 & 4.53 \\
\hline & 3452.89 & $17 \mathrm{Ni}$ & 1.0 & 0.11 & 3.68 \\
\hline 3461.9 & 3461.65 & $17 \mathrm{Ni}$ & 3.2 & 0.03 & 3.59 \\
\hline 3466.0 & 3465.86 & $6 \mathrm{Fe}$ & 0.52 & 0.11 & 3.67 \\
\hline 3472.0 & 3471.27 & $82 \mathrm{Fe}$ & & 2.21 & 5.77 \\
\hline & 3472.54 & $20 \mathrm{Ni}$ & 1.2 & 0.11 & 3.66 \\
\hline 3475.5 & 3475.45 & $6 \mathrm{Fe}$ & 0.64 & 0.09 & 3.64 \\
\hline 3476.5 & 3476.70 & $6 \mathrm{Fe}$ & 0.28 & 0.12 & 3.67 \\
\hline 3482.5 & 3483.01 & $24 \mathrm{Fe}$ & & 0.91 & 4.45 \\
\hline 3490.6 & 3490.58 & $6 \mathrm{Fe}$ & 0.58 & 0.05 & 3.59 \\
\hline 3492.2 & 3492.96 & $18 \mathrm{Ni}$ & 3.9 & 0.11 & 3.64 \\
\hline 3497.8 & 3497.84 & $6 \mathrm{Fe}$ & 0.19 & 0.11 & 3.64 \\
\hline 3501.7 & 3502.28 & $21 \mathrm{Co}$ & 11 & 0.43 & 3.95 \\
\hline 3505.0 & 3506.31 & $21 \mathrm{Co}$ & 9.4 & 0.51 & 4.03 \\
\hline 3512.5 & 3512.64 & $21 \mathrm{Co}$ & 7.4 & 0.58 & 4.09 \\
\hline 3514.0 & 3513.82 & $24 \mathrm{Fe}$ & 1.7 & 0.86 & 4.37 \\
\hline 3514.8 & 3515.05 & $19 \mathrm{Ni}$ & 4.5 & 0.11 & 3.62 \\
\hline 3522.0 & 3521.26 & $24 \mathrm{Fe}$ & 1.7 & 0.91 & 4,42 \\
\hline 3526.0 & 3524.54 & $18 \mathrm{Ni}$ & 4.6 & 0.03 & 3.53 \\
\hline & 3526.04 & $6 \mathrm{Fe}$ & 0.13 & 0.09 & 3.59 \\
\hline 3557.5 & 3558.52 & $24 \mathrm{Fe}$ & 3.5 & 0.99 & 4.45 \\
\hline 3566.0 & 3565.38 & $24 \mathrm{Fe}$ & 7.8 & 0.95 & 4.42 \\
\hline & 3566.37 & $36 \mathrm{Ni}$ & 6.4 & 0.42 & 3.88 \\
\hline 3560.5 & 3570.10 & $24 \mathrm{Fe}$ & 18 & 0.91 & 4.37 \\
\hline 3578.5 & 3578.69 & $4 \mathrm{Cr}$ & 8.3 & 0.00 & 3.45 \\
\hline 3581.2 & 3581.20 & $23 \mathrm{Fc}$ & 23 & 0.86 & 4.30 \\
\hline 3585.5 & 3585.32 & $23 \mathrm{Fe}$ & 1.7 & 0.95 & 4.40 \\
\hline & 3585.71 & $23 \mathrm{Fe}$ & 1.3 & 0.91 & 4.35 \\
\hline 3586.8 & 3586.99 & $23 \mathrm{Fe}$ & 2.0 & 0.99 & 4.43 \\
\hline 3589.6 & 3589.11 & $23 \mathrm{Fe}$ & 0.26 & 0.86 & 4.29 \\
\hline 3593.2 & 3593.49 & $4 \mathrm{Cr}$ & 7.0 & 0.00 & 3.43 \\
\hline 3605.5 & 3605.33 & $4 \mathrm{Cr}$ & 5.2 & 0.00 & 3.42 \\
\hline & 3605.46 & $294 \mathrm{Fe}$ & 51 & 2.72 & 6.14 \\
\hline 3606.7 & 3606.68 & $294 \mathrm{Fc}$ & 65 & 2.68 & 6.10 \\
\hline 3608.9 & 3608.86 & $23 \mathrm{Fe}$ & 10 & 1.01 & 4.43 \\
\hline 3618.8 & 3618.77 & $23 \mathrm{Fe}$ & 9.5 & 0.99 & 4.40 \\
\hline 3631.5 & 3631.46 & $23 \mathrm{Fe}$ & 8.6 & 0.95 & 4.35 \\
\hline $36+7.8$ & 3647.84 & $23 \mathrm{Fe}$ & 6.1 & 0.91 & 4.29 \\
\hline
\end{tabular}


TABLE 1.-Wavelength Identifications of Sporadic Meteor-Continued

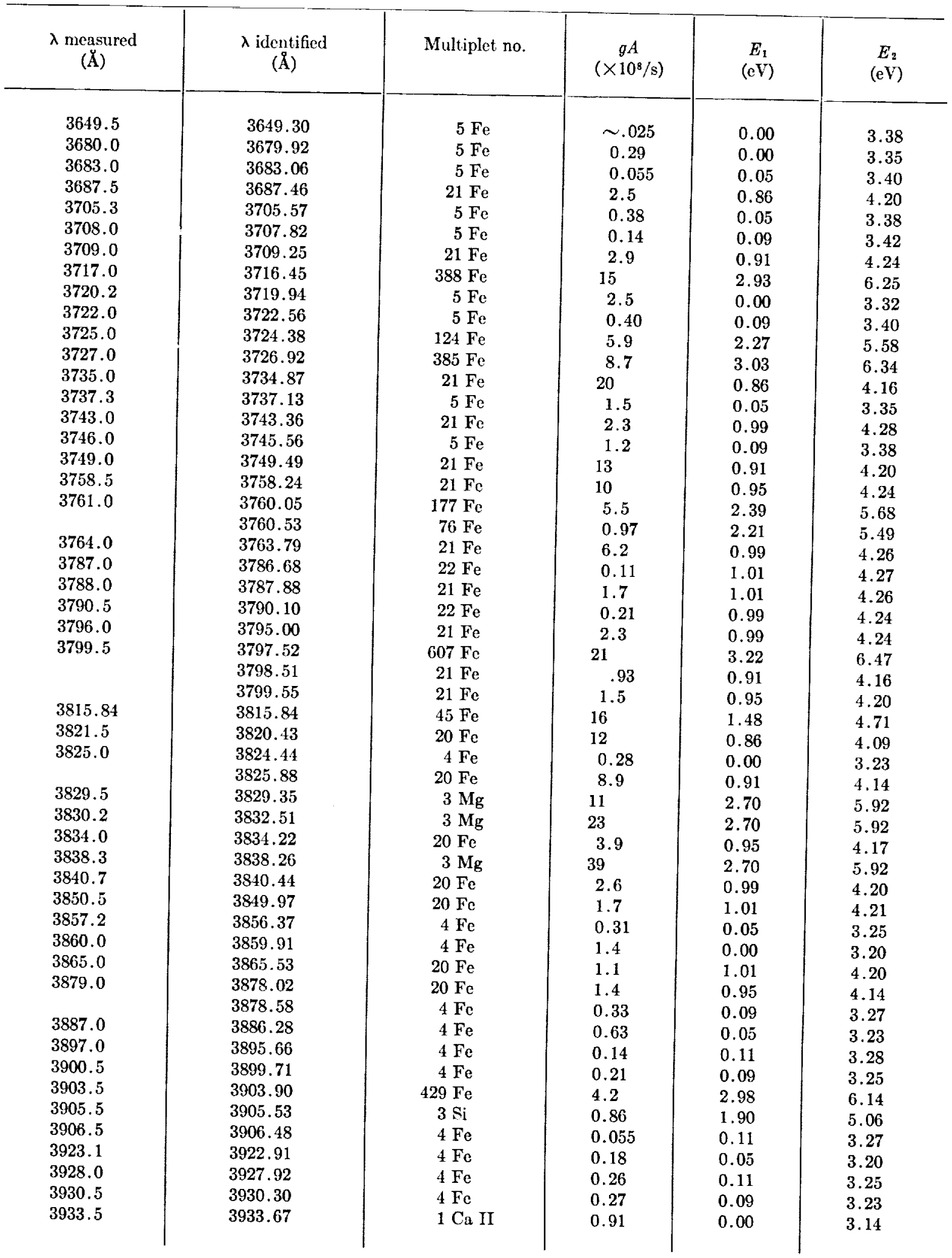


Tab1, 1.-Watelength Identifications of Sporadic Meteor-Continued

\begin{tabular}{|c|c|c|c|c|c|}
\hline $\begin{array}{c}\lambda \text { meastured } \\
(\AA)\end{array}$ & $\begin{array}{c}\lambda \text { identified } \\
(\AA)\end{array}$ & Multiplet no. & $\begin{array}{c}g A \\
\left(\times 10^{8 / 5}\right)\end{array}$ & $\begin{array}{c}E_{1} \\
(\mathrm{eV})\end{array}$ & $\begin{array}{c}E_{2} \\
(\mathrm{eV})\end{array}$ \\
\hline 3942.2 & 3942.44 & $364 \mathrm{Fe}$ & 2.5 & 2.83 & 5.96 \\
\hline 3969.3 & 3968.47 & $1 \mathrm{Ca} \mathrm{II}$ & 0.45 & 0.00 & 3.11 \\
\hline & 3969.26 & $43 \mathrm{Fc}$ & 4.4 & 1.48 & 4.59 \\
\hline 4004.5 & 4005.25 & $43 \mathrm{Fe}$ & 3.6 & 1.55 & 4.63 \\
\hline 4031.0 & 4030.76 & $2 \mathrm{Mn}$ & 1.4 & 0.00 & 3.06 \\
\hline 4033.0 & 4033.07 & $2 \mathrm{Mn}$ & 0.95 & 0.00 & 3.06 \\
\hline 4036.0 & 4034.49 & $2 \mathrm{Mn}$ & 0.54 & 0.00 & 3.06 \\
\hline 4045.8 & 4045.82 & $43 \mathrm{Fe}$ & 22 & 1.48 & 4.53 \\
\hline 4063.5 & 4063.60 & $43 \mathrm{Fc}$ & 9.9 & 1.55 & 4.59 \\
\hline 4071.5 & 4071.74 & $43 \mathrm{Fe}$ & 9.1 & 1.60 & 4.63 \\
\hline 4133.0 & 4132.06 & $43 \mathrm{Fe}$ & 2.7 & 1.60 & 4.59 \\
\hline 4143.9 & 4143.42 & $523 \mathrm{Fe}$ & 16 & 3.03 & 6.01 \\
\hline & 4143.87 & $43 \mathrm{Fe}$ & 2,9 & 1.55 & 4.53 \\
\hline 4202.0 & 4202.03 & $42 \mathrm{Fe}$ & 2.0 & 1.48 & 4.42 \\
\hline 4206.0 & 4206.70 & $3 \mathrm{Fe}$ & & 0.05 & 2.99 \\
\hline 4216.0 & 4216.19 & $3 \mathrm{Fe}$ & 0.0031 & 0.00 & 2.93 \\
\hline 4226.5 & 4226.73 & $2 \mathrm{Ca}$ & 1 & 0.00 & 2.92 \\
\hline 4251.0 & 4250.79 & $42 \mathrm{Fe}$ & 1.5 & 1.55 & 4.45 \\
\hline 4254.5 & 4254.35 & $1 \mathrm{Cr}$ & 2.0 & 0.00 & 2.90 \\
\hline 4257.8 & 4258,32 & $3 \mathrm{Fe}$ & & 0.09 & 2.99 \\
\hline 4271.8 & 4271.76 & $42 \mathrm{Fe}$ & 5.2 & 1.48 & 4.37 \\
\hline 4274.5 & 4274.80 & $1 \mathrm{Cr}$ & 1.5 & 0.00 & 2.89 \\
\hline 4290.0 & 4289.72 & $1 \mathrm{Cr}$ & 0.95 & 0.00 & 2.88 \\
\hline 4291.5 & 4291.66 & $3 \mathrm{Fe}_{\mathrm{e}}$ & & 0.09 & 2.99 \\
\hline 4293.5 & 4294,13 & $41 \mathrm{Fe}$ & 0.71 & 1.48 & 4.35 \\
\hline 4299.0 & 4299.24 & $152 \mathrm{Fe}$ & 5.2 & 2.41 & 5.29 \\
\hline 4308.0 & 4307.91 & $42 \mathrm{Fe}$ & 5.9 & 1.55 & 4.42 \\
\hline 4325.8 & 4325.76 & $42 \mathrm{Fe}$ & 6.1 & 1.60 & 4.45 \\
\hline 4376.0 & 4375.93 & $2 \mathrm{Fe}$ & 0.0094 & 0.00 & 2.82 \\
\hline 4383.5 & 4383.55 & $41 \mathrm{Fe}$ & 7.7 & 1.48 & 4.29 \\
\hline 4404.75 & 4404.75 & $41 \mathrm{Fc}$ & 4.4 & 1.55 & 4.35 \\
\hline 4427.3 & 4427.31 & $2 \mathrm{Fe}$ & 0.0091 & 0.05 & 2.84 \\
\hline 4461.65 & 4461.65 & $2 \mathrm{Fe}$ & 0.0052 & 0.09 & 2.85 \\
\hline 4482.17 & 4482.17 & $2 \mathrm{Fe}$ & 0.0053 & 0.11 & 2.86 \\
\hline
\end{tabular}

\section{TAURID METEOR}

The spectrum of the Taurid meteor is shown in figure 2. This spectrum was obtained during the night of November 4, 1969. The metror occurred at $22: 25$ hours, local time. $A$ beginning height of $100 \mathrm{~km}$ was assumed for the metcor. This hright is in general agreement with beginning heights of meteors of similar brightness and velocity (Jacchia et al., 1967). A torminal height of $68 \mathrm{~km}$ was calculated from the beginning height and relevant geometry. The metcor began $8^{\circ}$ from the radiant, and the trajectory made an angle of $10^{\circ}$ with the optical axis of the spcetro- graph. Hence the meteor was nearly "head-on" and was very favorable for photographic recording. An absolute meteor magnitude of -4 was obtained for this meteor by comparing its intensity in the blue and near ultraviolet region with that of an artificial moteor (Harvey, 1967a). The Taurid meteor speetrum was recorded on the same spectrograph as the sporadic meteor spectrum. However, the Taurid spectrum (as well as the Geminid and Perseid spectra) were recorded on "metcor recording film SO-153," which is similar to extended red emulsion type 2485. That is, the spectrum covers the wavelength intorval $3100 \AA$ to $7000 \AA$. Two hundred 
Ist ORDER SPECTRUM

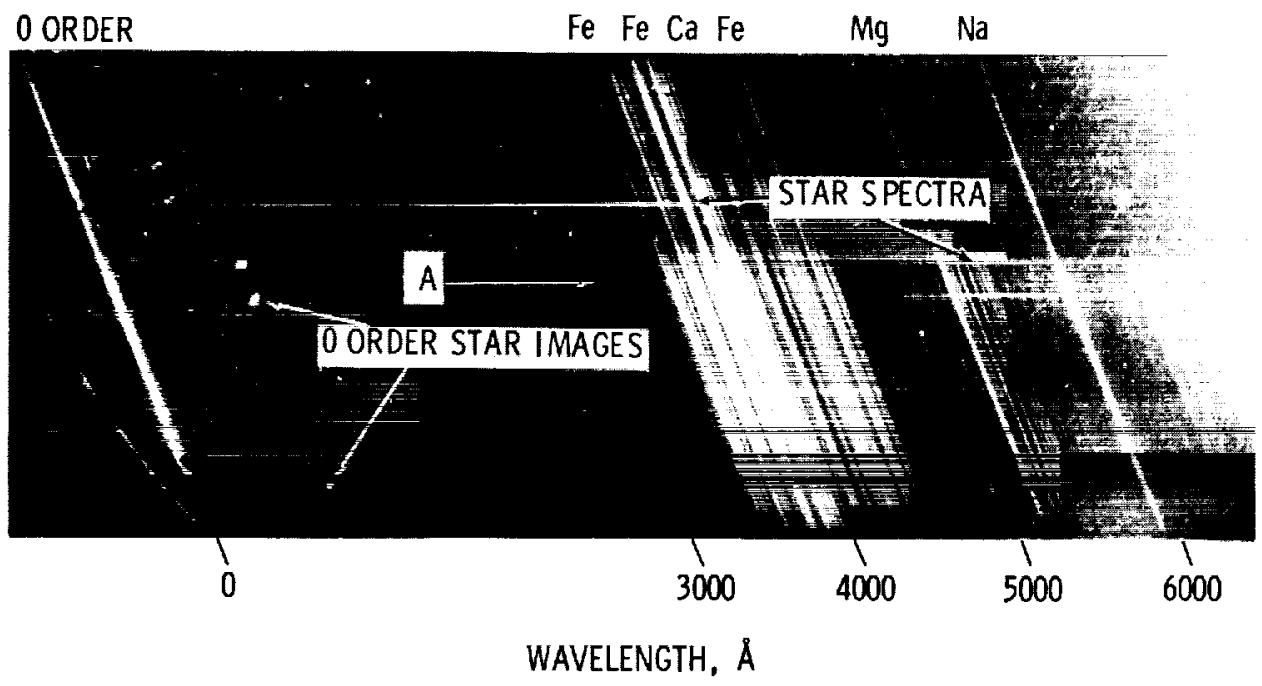

Figure 2.-Enlargement of a spectrogram of a Taurid meteor.

and thirty-seven of the strongest lines at position $A$ in the spectrum have been identified and are listed in table 2 . The strongest features in the spectrum are multiplets 4,5 , and 20 of neutral iron, 2 of neutral magnesium, and the sodium $D$ lincs. The spectrum suffers from mutliple zero-order star images and a dense and nonuniform background.

\section{GEMINID METEOR}

The spectrum of the Geminid meteor is shown in figure 3 . This spectrum was obtained on the night of December 12, 1969. The meteor occurred at approximately 03:40, local time. The meteor began $51^{\circ}$ from the Geminid radiant and ended $63^{\circ}$ from the radiant. An estimated beginning height of $100 \mathrm{~km}$ was agnin used from which a terminal height of $68 \mathrm{~km}$ was computed. An absolute meteor magnitude of $-\bar{j}$ was obtained for this meteor by the same method used for the Tiurid. The Geminid spectrum was recorded by the same spectrograph as the Taurid spectrum. One hundred and fifty-seren of the strongest lines at position $A$ have been identified in the preliminary wavelength analysis and are listed in table 3 . The strongest features in the spectrum are multiplets 4,5 , and 20 of neutral iron, 2 of magnesium, and the sodium $D$ lines. This Geminid spectrum is a "clean" spectrum in that it is not degraded by star images, poor resolution, or nonuniform background.

\section{PERSEID METEOR}

The spectrum of the Perscid meteor is shown in figure 4. The spectrum was obtained on the night of August 12, 1969. The meteor occurred between 02:30 and 4:00, local time. The meteor was approximately $90^{\circ}$ from the radiant and traveling nearly perpendicular to the optical axis of the spectrograph when photographed. A beginning height of $105 \mathrm{~km}$ was obtained from the maximum of the auroral green line. A terminal height of $92 \mathrm{~km}$ was calculated. A maximum brightness of -9 absolute meteor magnitude was obtained for this Perseid meteor by the same method as used for the Taurid and Geminid meteors. This spectrum was also recorded on the same spectrograph which recorded the other three spectra. As can be scen in figure 4 , the upper third of the spectrum is similar to that of the Geminid and Taurid meteors. However, in the latter part of the spectrum, the ionic lines of calcium, magnesium, and silicon become dominant. Ninety-four of the strongest lines at position $A$ hare been identified in the preliminary wavelength analysis and are listed in table 4. The entries of table 4 are from the same source as tables 1 to 3 except that the $g A$ values for $\mathrm{Si}$ 
TARLF 2.-Wavelength Identificalions of Taurid Meteor

\begin{tabular}{|c|c|c|c|c|c|}
\hline $\begin{array}{c}\lambda \text { measured } \\
(\AA)\end{array}$ & $\begin{array}{c}\lambda \text { identified } \\
(\AA)\end{array}$ & Multiplet no. & $\begin{array}{c}g A \\
\left(\times 10^{8} / \mathrm{s}\right)\end{array}$ & $\begin{array}{c}E_{1} \\
(\mathrm{eV})\end{array}$ & $\begin{array}{c}E_{2} \\
(\mathrm{eV})\end{array}$ \\
\hline \multirow[t]{4}{*}{3098} & 3099.97 & $28 \mathrm{Fc}$ & 8.6 & 0.91 & 4.89 \\
\hline & 3100.30 & $28 \mathrm{Fe}$ & 3.9 & 0.99 & 4.97 \\
\hline & 3100.67 & $28 \mathrm{Fe}$ & 3.6 & 0.95 & 4.93 \\
\hline & 3101.55 & $25 \mathrm{Ni}$ & 4.6 & 0,11 & 4.09 \\
\hline 3135 & 3134.11 & $25 \mathrm{Ni}$ & 5.8 & 0.21 & 4.15 \\
\hline 3184 & 3184.90 & $7 \mathrm{Fe}$ & 0.086 & 0.05 & 3.93 \\
\hline \multirow[t]{2}{*}{3193} & 3191.66 & $8 \mathrm{Fe}$ & 0.087 & 0.00 & 3.87 \\
\hline & 3193.21 & $7 \mathrm{Fe}$ & 0.14 & 0.00 & 3.86 \\
\hline 3200 & 3199.92 & $27 \mathrm{Ti}$ & 14 & 0.05 & 3.90 \\
\hline 3214 & 3214.40 & $7 \mathrm{Fe}$ & 0.086 & 0.09 & 3.93 \\
\hline 3224 & 3225.79 & $155 \mathrm{Fe}$ & 107 & 2.39 & 6.21 \\
\hline 3233 & 3234.52 & $2 \mathrm{Ti}$ II & 16 & 0.05 & 3.86 \\
\hline 3244 & 3243.06 & $22 \mathrm{Ni}$ & 0.62 & 0.03 & 3.83 \\
\hline 3251 & 3251.24 & $93 \mathrm{Fc}$ & & 2.19 & 5.98 \\
\hline 3254 & 3254.36 & $620 \mathrm{Fe}$ & & 3.25 & 7.05 \\
\hline 3265 & 3265.62 & $91 \mathrm{Fe}$ & 18 & 2.17 & 5.95 \\
\hline 3270 & 3271.00 & $91 \mathrm{Fe}$ & 22 & 2.19 & 5.96 \\
\hline 3284 & 3281.59 & $91 \mathrm{Fe}$ & & 2.19 & 5.95 \\
\hline \multirow[t]{2}{*}{3291} & 3290.99 & $95 \mathrm{Fe}$ & & 2.21 & 5.96 \\
\hline & 3202.50 & $91 \mathrm{Fe}$ & & 2.21 & 5.96 \\
\hline \multirow[t]{2}{*}{3301} & 3302.32 & $2 \mathrm{Na}$ & 0.65 & 0.00 & 3.74 \\
\hline & 3302.99 & $2 \mathrm{Na}$ & 0.33 & 0.00 & 3.74 \\
\hline \multirow{2}{*}{3306} & 3306.0 & $91 \mathrm{Fe}$ & 38 & 2.91 & 5.92 \\
\hline & 3306.4 & $91 \mathrm{Fe}$ & 40 & 2.21 & 5.95 \\
\hline 3320 & 3320.26 & $9 \mathrm{Ni}$ & 0.39 & 0.16 & 3.88 \\
\hline 3332 & 3331.62 & $191 \mathrm{Fe}$ & & 2.42 & 6.13 \\
\hline 3335 & 3334.22 & $190 \mathrm{Fe}$ & & 2.42 & 6.12 \\
\hline 3342 & $33+1.88$ & $24 \mathrm{Ti}$ & 13 & 0.00 & 3.69 \\
\hline \multirow[t]{4}{*}{$\begin{array}{l}3342 \\
3352\end{array}$} & 3344.51 & $11 \mathrm{Ca}$ & & 1.88 & 5.56 \\
\hline & 3350.21 & $11 \mathrm{Ca}$ & & 1.88 & 5.56 \\
\hline & 3350.36 & $11 \mathrm{Ca}$ & & 1.88 & 5.56 \\
\hline & $335+.64$ & $24 \mathrm{Ti}$ & 9.7 & 0.02 & 3.71 \\
\hline \multirow[t]{2}{*}{3363} & 3361.92 & $11 \mathrm{Ca}$ & & 1.89 & 5.56 \\
\hline & 3362.13 & $11 \mathrm{Ca}$ & & 1.89 & 5.56 \\
\hline \multirow[t]{2}{*}{3365} & 3365.77 & $38 \mathrm{Ni}$ & 0.63 & 0.42 & 4.09 \\
\hline & 3366.17 & $8 \mathrm{Ni}$ & 0.35 & 0.16 & 3.83 \\
\hline 3375 & 3374.22 & $17 \mathrm{Ni}$ & 0.20 & 0.03 & 3.68 \\
\hline \multirow[t]{2}{*}{338.1} & 3383.69 & $85 \mathrm{Fe}$ & & 2.19 & 5.84 \\
\hline & 3383.08 & $83 \mathrm{Fo}$ & 0.1 & 2.17 & 5.81 \\
\hline 3390 & 3399.34 & $85 \mathrm{Fo}^{\circ}$ & 25 & 2.19 & 5.82 \\
\hline 3404 & 3404.36 & $83 \mathrm{Fe}$ & 17 & 2.19 & 5.81 \\
\hline 3407 & 3407.46 & $83 \mathrm{Fc}$ & 33 & 2.17 & 5.79 \\
\hline 3414 & 3413.14 & $8 j \mathrm{Fe}$ & 26 & 2.19 & 5.80 \\
\hline 3424 & $3+2+.29$ & $81 \mathrm{Fe}$ & 17 & 2.17 & 5.77 \\
\hline \multirow[t]{2}{*}{3425} & 3426.39 & $82 \mathrm{Fe}$ & 7.4 & 2.19 & 5.79 \\
\hline & $3+26.64$ & $82 \mathrm{Fe}$ & 7.7 & 2.19 & 5.79 \\
\hline 3429 & $3+27.12$ & $81 \mathrm{Fe}$ & 34 & 2.17 & 5.77 \\
\hline \multirow[t]{2}{*}{3440} & 3440.61 & $6 \mathrm{FC}$ & 2.8 & 0.00 & 3.59 \\
\hline & 3140.99 & $6 \mathrm{Fo}$ & 0.64 & 0.05 & 3.64 \\
\hline \multirow[t]{2}{*}{3450} & 3450.33 & $\$ 2 \mathrm{Fc}$ & 8.9 & 2.21 & 5.79 \\
\hline & 3451.92 & $\mathrm{Sl} \mathrm{Fo}$ & 8.8 & 2.21 & 5.79 \\
\hline 3460 & 3458.47 & $19 \mathrm{Ni}$ & 4.0 & 0.21 & 3.78 \\
\hline 3462 & 3461.65 & $17 \mathrm{Ni}$ & 3.2 & 0.03 & 3.59 \\
\hline
\end{tabular}


TABLE 2.-Wavelength Identifications of Taurid Meteor-Continued

\begin{tabular}{|c|c|c|c|c|c|}
\hline $\begin{array}{c}\lambda \text { measured } \\
(\AA)\end{array}$ & $\begin{array}{c}\lambda \text { identified } \\
(\AA)\end{array}$ & Multiplet no. & $\left(\begin{array}{c}g A \\
\left(\times 10^{8} / \mathrm{s}\right)\end{array}\right.$ & $\begin{array}{c}E_{1} \\
(\mathrm{eV})\end{array}$ & $\begin{array}{c}E_{2} \\
(\mathrm{eV})\end{array}$ \\
\hline 3466 & 3465.86 & $6 \mathrm{Fe}$ & 0.52 & 0.11 & 3.67 \\
\hline 3476 & 3475.45 & $6 \mathrm{Fe}$ & 0.64 & 0.09 & 3.64 \\
\hline \multirow[t]{2}{*}{3491} & 3490.58 & $6 \mathrm{Fe}$ & 0.58 & 0.05 & 3.59 \\
\hline & 3492.96 & $18 \mathrm{Ni}$ & 3.9 & 0.11 & 3.64 \\
\hline 3499 & 3497.84 & $6 \mathrm{Fe}$ & 0.19 & 0.11 & 3.64 \\
\hline 3514 & 3513,82 & $24 \mathrm{Fe}$ & 1.7 & 0.86 & 4.37 \\
\hline 3526 & 3526.04 & $6 \mathrm{Fe}$ & 0.13 & 0.09 & 3.59 \\
\hline 3534 & 3536.56 & $326 \mathrm{Fe}$ & 56 & 2.86 & 6.35 \\
\hline \multirow[t]{2}{*}{3541} & 3541.09 & $326 \mathrm{Fe}$ & 65 & 2.84 & 6.32 \\
\hline & 3542.08 & $326 \mathrm{Fe}$ & 61 & 2.85 & 6.34 \\
\hline 3554 & 3554.93 & $326 \mathrm{Fe}$ & 73 & 2.82 & 6.29 \\
\hline 3560 & 3558.52 & $24 \mathrm{Fe}_{\mathrm{e}}$ & 3.5 & 0.99 & 4.45 \\
\hline 3565 & 3565.38 & $24 \mathrm{Fe}$ & 7.8 & 0.95 & 4.42 \\
\hline 3570 & 3570.10 & $24 \mathrm{Fe}$ & 18 & 0.91 & 4.37 \\
\hline 3578 & 3578.69 & $4 \mathrm{Cr}$ & 8.3 & 0.00 & 3.45 \\
\hline 3581 & 3581.20 & $23 \mathrm{Fe}$ & 23 & 0.86 & 4.30 \\
\hline 3586 & 3586.99 & $23 \mathrm{Fe}$ & 2.0 & 0.99 & 4.43 \\
\hline 3588 & 3589.11 & $23 \mathrm{Fe}$ & 0.26 & 0.86 & 4.29 \\
\hline 3594 & 3594.64 & $322 \mathrm{Fe}$ & 21 & 2.84 & 6.27 \\
\hline \multirow[t]{2}{*}{3604} & 3603.21 & $295 \mathrm{Fe}$ & 33 & 2.68 & 6.11 \\
\hline & 3605.46 & $294 \mathrm{Fc}$ & 33 & 2.72 & 6.14 \\
\hline 3607 & 3606.68 & $294 \mathrm{Fe}$ & 65 & 2.68 & 6.10 \\
\hline 3609 & 3608.86 & $23 \mathrm{Fe}$ & 10 & 1.01 & 4.43 \\
\hline 3611 & 3610.16 & $321 \mathrm{Fe}$ & 48 & 2.80 & 6.21 \\
\hline 3619 & 3618.77 & $23 \mathrm{Fe}$ & 9.5 & 0.99 & 4.40 \\
\hline 3620 & 3619.39 & $35 \mathrm{Ni}$ & 7.5 & 0.42 & 3.83 \\
\hline \multirow[t]{2}{*}{3632} & 3631.46 & $23 \mathrm{Fe}$ & 8.6 & 0.95 & 4.35 \\
\hline & 3632.04 & $496 \mathrm{Fe}$ & 26 & 3.06 & 6.46 \\
\hline 3639 & 3638.30 & $294 \mathrm{Fe}$ & 28 & 2.75 & 6.14 \\
\hline 3646 & 3645.82 & $496 \mathrm{Fe}$ & 20 & 3.10 & 6.48 \\
\hline 3648 & 3647.84 & $23 \mathrm{Fe}$ & 6.1 & 0.91 & 4.29 \\
\hline 3659 & 3659.52 & $180 \mathrm{Fe}$ & 9.9 & 2.44 & 5.82 \\
\hline 3671 & 3669.52 & $291 \mathrm{Fe}$ & 32 & 2.72 & 6.08 \\
\hline 3680 & 3679.92 & $5 \mathrm{Fe}$ & 0.29 & 0.00 & 3.35 \\
\hline 3683 & 3683.06 & $5 \mathrm{Fe}$ & 0.055 & 0.05 & 3.40 \\
\hline 3687 & 3687.46 & $21 \mathrm{Fe}$ & 2.5 & 0.86 & 4.20 \\
\hline 3694 & 3794.01 & $394 \mathrm{Fe}$ & 72 & 3.03 & 6.37 \\
\hline 3700 & 3701.09 & $385 \mathrm{Fe}$ & 85 & 2.99 & 6.32 \\
\hline 3706 & 3705.57 & $5 \mathrm{Fe}$ & 0.38 & 0.05 & 3.38 \\
\hline 3708 & 3707.82 & $5 \mathrm{Fe}$ & 0.14 & 0.09 & 3.42 \\
\hline 3720 & 3719.94 & $5 \mathrm{Fe}$ & 2.5 & 0.00 & 3.32 \\
\hline 3723 & 3722.56 & $5 \mathrm{Fe}$ & 0.40 & 0.09 & 3.40 \\
\hline \multirow[t]{2}{*}{3733} & 3733.32 & $5 \mathrm{Fe}$ & 0.36 & 0.11 & 3.42 \\
\hline & 3634.87 & $21 \mathrm{Fe}$ & 20 & 0.86 & 4.16 \\
\hline 3737 & 3737.13 & $5 \mathrm{Fe}$ & 1.5 & 0.05 & 3.35 \\
\hline \multirow[t]{2}{*}{3747} & 3745.56 & $5 \mathrm{Fe}$ & 1.2 & 0.09 & 3.38 \\
\hline & 3745.90 & $5 \mathrm{Fe}$ & 0.31 & 0.12 & 3.42 \\
\hline \multirow[t]{2}{*}{3749} & 3748.26 & $5 \mathrm{Fe}$ & 0.71 & 0.11 & 3.40 \\
\hline & 3749.49 & $21 \mathrm{Fe}$ & 13 & 0.91 & 4.20 \\
\hline 3759 & 3758.24 & $21 \mathrm{Fe}$ & 10 & 0.95 & 4.24 \\
\hline \multirow[t]{2}{*}{3764} & 3763.79 & $21 \mathrm{Fe}$ & 6.2 & 0.99 & 4.26 \\
\hline & 3765.54 & $608 \mathrm{Fe}$ & 50 & 3.22 & 6.50 \\
\hline 3768 & 3767.19 & $21 \mathrm{Fe}$ & 4.6 & 1.01 & 4.28 \\
\hline
\end{tabular}


SPECTRAL ANALYSIS OF FOUR METEORS

TABLE 2.-Wavelength Identifications of Taurid Meteor-Continued

\begin{tabular}{|c|c|c|c|c|c|}
\hline$\lambda$ mensured & $\begin{array}{c}\lambda \text { identified } \\
(\AA)\end{array}$ & Multiplet no. & $\begin{array}{c}g A \\
\left(\times 10^{8} / \mathrm{s}\right)\end{array}$ & $\begin{array}{c}E_{1} \\
(\mathrm{eV})\end{array}$ & $\begin{array}{c}E_{2} \\
(\mathrm{eV})\end{array}$ \\
\hline \multirow[t]{3}{*}{3775} & 3774.82 & $73 \mathrm{Fe}$ & 0.59 & 2.21 & 5.48 \\
\hline & 3775.57 & $33 \mathrm{Ni}$ & 0.57 & 0.42 & 3.69 \\
\hline & 3776.45 & $74 \mathrm{Fe}$ & 0.53 & 2.17 & 5.43 \\
\hline 3786 & 3786.68 & $22 \mathrm{Fe}$ & & 1.01 & 4.27 \\
\hline 3791 & 3790.10 & $22 \mathrm{Fe}$ & 0.21 & 0.99 & 4.24 \\
\hline 3795 & 3795.00 & $21 \mathrm{Fe}$ & 2.3 & 0.99 & 4.24 \\
\hline \multirow[t]{2}{*}{3797} & 3798.51 & $21 \mathrm{Fe}$ & 0.93 & 0.91 & 4.16 \\
\hline & 3799.55 & $21 \mathrm{Fe}$ & 1.5 & 0.95 & 4.20 \\
\hline 3813 & 3812.96 & $22 \mathrm{Fe}$ & 1.0 & 0.95 & 4.19 \\
\hline 3817 & 3815.84 & $45 \mathrm{Fe}$ & 16 & 1.48 & 4.71 \\
\hline .3821 & 3820.43 & $20 \mathrm{Fe}$ & 12 & 0.86 & 4.09 \\
\hline \multirow[t]{2}{*}{3825} & 3824.44 & $4 \mathrm{Fc}$ & 0.28 & 0.00 & 3.32 \\
\hline & 3825.88 & $20 \mathrm{Fe}$ & 8.9 & 0.91 & 4.14 \\
\hline \multirow[t]{3}{*}{3831} & 3827.82 & $45 \mathrm{Fe}$ & 15 & 1.55 & 4.77 \\
\hline & 3829.35 & $3 \mathrm{Mg}$ & 11 & 2.70 & 5.92 \\
\hline & 3832.51 & $3 \mathrm{Mg}$ & 23 & 2.70 & 5.92 \\
\hline 3838 & 3838.26 & $3 \mathrm{Mg}$ & 39 & 2.70 & 5.92 \\
\hline \multirow[t]{2}{*}{3849} & 3849.97 & $20 \mathrm{Fe}$ & 1.7 & 1.01 & 4.21 \\
\hline & 3850.82 & $22 \mathrm{Fe}$ & 0.22 & 0.99 & 4.19 \\
\hline \multirow{2}{*}{3855} & 3856.37 & $4 \mathrm{Fe}$ & 0.31 & 0.05 & 3.25 \\
\hline & 3856.37 & $1 \mathrm{Si} \mathrm{II}$ & & 6.83 & 10.03 \\
\hline 3860 & 3859.91 & $4 \mathrm{Fe}$ & 1.4 & 0.00 & 3.20 \\
\hline 3862 & 3862.59 & $1 \mathrm{Si}$ II & & 6.83 & 10.03 \\
\hline 3874 & 3873.76 & $175 \mathrm{Fe}$ & 2.8 & 2.42 & 5.61 \\
\hline \multirow[t]{2}{*}{3878} & 3878.02 & $20 \mathrm{Fe}$ & 1.4 & 0.95 & 4.14 \\
\hline & 3878.58 & $4 \mathrm{Fe}$ & 0.33 & 0.09 & 3.27 \\
\hline 3886 & 3886.28 & $4 \mathrm{Fe}$ & 0.63 & 0.05 & 3.23 \\
\hline 3896 & 3895.66 & $4 \mathrm{Fe}$ & 0.14 & 0.11 & 3.28 \\
\hline 3900 & 3899.71 & $4 \mathrm{Fe}$ & 0.21 & 0.09 & 3.25 \\
\hline \multirow[t]{2}{*}{3905} & 3905.53 & $3 \mathrm{Si}$ & 0.20 & 1.90 & 5.06 \\
\hline & 3906.48 & $4 \mathrm{Fe}$ & 0.055 & 0.11 & 3.27 \\
\hline 3921 & 3920.26 & $4 \mathrm{Fe}$ & 0,14 & 0.12 & 3.27 \\
\hline 3922 & 3922.91 & $4 \mathrm{Fe}$ & 0.18 & 0.05 & 3.20 \\
\hline \multirow[t]{2}{*}{3929} & 3927.92 & $4 \mathrm{Fe}$ & 0.26 & 0.11 & 3.25 \\
\hline & 3930.30 & $4 \mathrm{Fe}$ & 0.27 & 0.09 & 3.23 \\
\hline 3936 & 3935.82 & $362 \mathrm{Fe}$ & 3.5 & 2.82 & 5.96 \\
\hline 3940 & 3940.88 & $20 \mathrm{Fe}$ & 0.041 & 0.05 & 4.09 \\
\hline 3944 & 3944,03 & $1 \mathrm{Al}$ & 0.66 & 0.00 & 3.13 \\
\hline 3956 & 3956.68 & $278 \mathrm{Fc}$ & 9.1 & 2.68 & 5.80 \\
\hline 3969.3 & 3969.26 & $43 \mathrm{Fe}$ & 4.4 & 1.48 & 4.59 \\
\hline \multirow[t]{2}{*}{4000} & 3997.40 & $278 \mathrm{Fe}$ & 11 & 2.72 & 5.80 \\
\hline & 3998.06 & $276 \mathrm{Fe}$ & 3.7 & 2.68 & 5.77 \\
\hline 4005 & 4005.25 & $43 \mathrm{Fe}$ & 3.6 & 1.55 & 4.63 \\
\hline 4010 & 4009.72 & $72 \mathrm{Fe}_{\mathrm{e}}$ & 1.4 & 2.21 & 5.29 \\
\hline \multirow[t]{3}{*}{4030} & 4030.76 & $2 \mathrm{Mn}$ & 1.4 & 0.00 & 3.06 \\
\hline & 4033.07 & $2 \mathrm{Mn}$ & 0.95 & 0.00 & 3.06 \\
\hline & 4034.49 & $2 \mathrm{Mn}$ & 0.54 & 0.00 & 3.06 \\
\hline 4040 & 4041.36 & $5 \mathrm{Mn}$ & 31 & 2.11 & 5.16 \\
\hline 4045 & 4045.82 & $43 \mathrm{Fe}$ & 22 & 1.48 & 4.53 \\
\hline 4060 & 4058.93 & $5 \mathrm{Mn}$ & 7.4 & 2.17 & 5.21 \\
\hline 4063 & 4063.60 & $43 \mathrm{Fe}$ & 9.9 & 1.55 & 4.59 \\
\hline 4071 & 4071.74 & $43 \mathrm{Fe}$ & 9.1 & 1.60 & 4.63 \\
\hline 4082 & 4082.94 & $5 \mathrm{Mn}$ & 7.1 & 2.17 & 5.19 \\
\hline
\end{tabular}


TABle 2.-Wavelength Identifications of Taurid Meteor-Continued

\begin{tabular}{|c|c|c|c|c|c|}
\hline $\begin{array}{c}\lambda \text { measured } \\
(\AA)\end{array}$ & $\begin{array}{l}\lambda \text { identified } \\
(\AA)\end{array}$ & Multiplet no. & $\begin{array}{c}g A \\
\left(\times 10^{\mathrm{g}} / \mathrm{s}\right)\end{array}$ & $\begin{array}{c}E_{1} \\
(\mathrm{eV})\end{array}$ & $\begin{array}{c}E_{2} \\
(\mathrm{eV})\end{array}$ \\
\hline & 4083.63 & $5 \mathrm{Mn}$ & 6.9 & 2.15 & 5.18 \\
\hline 4100 & 4100.75 & $18 \mathrm{Fc}$ & & 0.86 & 3.86 \\
\hline 4108 & 4107.49 & $354 \mathrm{Fc}_{\mathrm{c}}$ & 5.6 & 2.82 & 5.82 \\
\hline 4120 & 4118.55 & $801 \mathrm{Fe}$ & 33 & 3.56 & 6.55 \\
\hline 4132 & 4132.06 & $43 \mathrm{Fe}$ & 2.7 & 1.60 & 4.59 \\
\hline \multirow[t]{2}{*}{4140} & 4139.93 & $18 \mathrm{Fe}$ & & 0.99 & 3.97 \\
\hline & 4143.87 & $43 \mathrm{Fr}$ & 2.9 & 1.55 & 4.53 \\
\hline \multirow[t]{2}{*}{4155} & 4154.50 & $355 \mathrm{Fe}$ & 5.3 & 2.82 & 5.79 \\
\hline & 4156.80 & $354 \mathrm{Fe}$ & 5.3 & 2.82 & 5.79 \\
\hline 4172 & 4172.75 & $19 \mathrm{Fe}$ & & 0.95 & 3.91 \\
\hline 1178 & 4177.60 & $18 \mathrm{Fe}$ & & 0.91 & 3.86 \\
\hline \multirow[t]{2}{*}{4189} & 4187.04 & $152 \mathrm{Fe}$ & 6.9 & 2.44 & 5.39 \\
\hline & 4187.80 & $152 \mathrm{Fe}$ & 6.5 & 2.41 & 5.36 \\
\hline 4201 & 4202.03 & $42 \mathrm{Fe}$ & 2.0 & 1.48 & 4.42 \\
\hline 4206 & 4206.70 & $3 \mathrm{Fe}$ & & 0.05 & 2.99 \\
\hline 4215 & 4216.19 & $3 \mathrm{Fe}$ & 0.0031 & 0.00 & 2.93 \\
\hline 4226.7 & 4226.73 & $2 \mathrm{Ca}$ & 1 & 0.00 & 2.92 \\
\hline 4235 & 4235.94 & $152 \mathrm{Fe}$ & 7.9 & 2.41 & 5.33 \\
\hline 4240 & 4238.82 & $693 \mathrm{Fe}$ & 9.4 & 3.38 & 6.29 \\
\hline 4251 & 4250.79 & $42 \mathrm{Fe}$ & 1.5 & 1.55 & 4.45 \\
\hline 4253 & 4254.35 & $1 \mathrm{Cr}$ & 2.0 & 0.00 & 5.36 \\
\hline 4261 & 4260.48 & $152 \mathrm{Fe}$ & 15 & 2.39 & 5.29 \\
\hline 4272 & 4271.76 & $42 \mathrm{Fe}$ & 5.2 & 1.48 & 4.37 \\
\hline 4276 & 4274.80 & $1 \mathrm{Cr}$ & 1.5 & 0.00 & 2.89 \\
\hline 4283 & 4282.41 & $71 \mathrm{Fe}$ & 2.0 & 2.17 & 5.05 \\
\hline \multirow[t]{2}{*}{4291} & 4289.72 & $1 \mathrm{Cr}$ & 0.95 & 0.00 & 2.88 \\
\hline & 4291.47 & $3 \mathrm{Fe}$ & & 0.09 & 2.99 \\
\hline 4300 & 4299.24 & $152 \mathrm{Fe}$ & 5.2 & 2.41 & 5.29 \\
\hline 4308 & 4307.91 & $42 \mathrm{Fe}$ & 5.9 & 1.55 & 4.42 \\
\hline 4325 & 4325.76 & $42 \mathrm{Fe}$ & 6.1 & 1.60 & 4.45 \\
\hline 4338 & 4337.05 & $42 \mathrm{Fe}$ & 0.23 & 1.55 & 4.40 \\
\hline 4354 & 4352.74 & $71 \mathrm{Fe}$ & 1 & 2.21 & 5.05 \\
\hline 4368 & 4368.30 & 50 & & 9.48 & 12.31 \\
\hline 4376 & 4375.93 & $2 \mathrm{Fe}$ & 0.0094 & 0.00 & 2.82 \\
\hline 4383 & 4383.55 & $41 \mathrm{Fe}$ & 7.7 & 1.48 & 4.29 \\
\hline 4405 & 4404.75 & $41 \mathrm{Fe}$ & 4.4 & 1.55 & 4.35 \\
\hline 4416 & 4415.12 & $41 \mathrm{Fe}$ & 2.8 & 1.60 & 4.40 \\
\hline 4427 & 4427.31 & $2 \mathrm{Fe}$ & 0.0099 & 0.05 & 2.84 \\
\hline \multirow[t]{2}{*}{4454} & 4454.78 & $4 \mathrm{Ca}$ & 7.5 & 1.89 & 4.66 \\
\hline & 4455.89 & $4 \mathrm{Ca}$ & 0.97 & 1.89 & 4.66 \\
\hline \multirow[t]{2}{*}{4462} & 4461.65 & $2 \mathrm{Fe}$ & 0.0052 & 0.09 & 2.85 \\
\hline & 4462.05 & $28 \mathrm{Mn}$ & 16 & 3.06 & 5.83 \\
\hline 4482 & 4482.17 & $2 \mathrm{Fe}$ & 0.0053 & 0.11 & 2.86 \\
\hline 4489 & 4489.74 & $2 \mathrm{Fe}$ & & 0.12 & 2.87 \\
\hline 4496 & 4494.57 & $68 \mathrm{Fe}$ & 1.2 & 2.19 & 4.93 \\
\hline \multirow[t]{2}{*}{4529} & 4528.62 & $68 \mathrm{Fe}$ & 1.8 & 2.17 & 4.89 \\
\hline & 4531.15 & $39 \mathrm{Fe}$ & 0.076 & 1.48 & 4.20 \\
\hline 4570 & 4571.10 & $1 \mathrm{Mg}$ & & 0.00 & 2.70 \\
\hline 4581 & 4581.40 & $23 \mathrm{Ca}$ & 0.96 & 2.51 & 5.21 \\
\hline 4586 & 4585.87 & $23 \mathrm{Ca}$ & 1.5 & 2.51 & 5.21 \\
\hline 4601 & 4602.94 & $39 \mathrm{Fe}$ & 0.088 & 1.48 & 4.16 \\
\hline 4692 & 4691.41 & $409 \mathrm{Fe}$ & & 2.98 & 5.61 \\
\hline 4703 & 4702.98 & $11 \mathrm{Mg}$ & & 4.33 & 6.95 \\
\hline
\end{tabular}


Table 2.-Wavelength Identifications of Taurid Meteor-Continued

\begin{tabular}{|c|c|c|c|c|c|}
\hline $\begin{array}{c}\lambda \text { measured } \\
(\AA)\end{array}$ & $\begin{array}{c}\lambda \text { identified } \\
(\AA)\end{array}$ & Multiplet no. & $\begin{array}{c}g A \\
\left(\times 10^{8} / \mathrm{s}\right)\end{array}$ & $\begin{array}{c}E_{1} \\
(\mathrm{eV})\end{array}$ & $\begin{array}{c}E_{2} \\
(\mathrm{eV})\end{array}$ \\
\hline 4737 & 4736.78 & $554 \mathrm{Fe}$ & 2.5 & 3.20 & 5.80 \\
\hline \multirow{2}{*}{4761} & 4761.53 & $21 \mathrm{Mn}$ & & 2.94 & 5.53 \\
\hline & 4762.38 & $21 \mathrm{Mn}$ & 12 & 2.88 & 5.47 \\
\hline \multirow[t]{2}{*}{4768} & 4765.86 & $21 \mathrm{Mn}$ & & 2.93 & 5.52 \\
\hline & 4766.43 & $21 \mathrm{Mn}$ & 8.4 & 2.91 & 5.50 \\
\hline 4825 & 4823.52 & $16 \mathrm{Mn}$ & 4.0 & 2.31 & 4.87 \\
\hline 4861 & 4859.75 & $318 \mathrm{Fe}$ & 1.3 & 2.86 & 5.40 \\
\hline \multirow[t]{2}{*}{4872} & 4871.32 & $318 \mathrm{Fe}$ & 3.7 & 2.85 & 5.39 \\
\hline & 4872.15 & $318 \mathrm{Fe}$ & 2.2 & 2.87 & 5.40 \\
\hline 4878 & 4878.22 & $318 \mathrm{Fe}$ & 0.77 & 2.87 & 5.40 \\
\hline \multirow[t]{2}{*}{4892} & 4890.77 & $318 \mathrm{Fe}$ & 2.2 & 2.86 & 5.39 \\
\hline & 4891.50 & $318 \mathrm{Fe}$ & 4.7 & 2.84 & 5.36 \\
\hline 4905 & 4903.32 & $318 \mathrm{Fe}$ & 0.62 & 2.87 & 5.39 \\
\hline 4919 & 4919.00 & $318 \mathrm{Fe}$ & 2.9 & 2.85 & 5.36 \\
\hline 4920 & 4920.50 & $318 \mathrm{Fe}$ & 6.5 & 2.82 & 5.33 \\
\hline 4939 & 4938.82 & $318 \mathrm{Fe}$ & & 2.86 & 5.36 \\
\hline \multirow[t]{2}{*}{4958} & 4957.31 & $318 \mathrm{Fe}$ & 2.2 & 2.84 & 5.33 \\
\hline & 4957.61 & $318 \mathrm{Fe}$ & 6.4 & 2.80 & 5.29 \\
\hline 4967 & 4966.10 & $687 \mathrm{Fe}$ & & 3.32 & 5.80 \\
\hline 4988 & 4985.55 & $318 \mathrm{Fe}$ & & 2.85 & 5.33 \\
\hline 4995 & 4994.13 & $16 \mathrm{Fe}$ & & 0.91 & 3.38 \\
\hline 5006 & 5006.13 & $318 \mathrm{Fe}$ & 1.3 & 2.82 & 5.29 \\
\hline 5014 & 5012.07 & $16 \mathrm{Fe}$ & 0.0067 & 0.86 & 3.32 \\
\hline 5041 & 5041.76 & $36 \mathrm{Fe}$ & 0.023 & 1.48 & 3.93 \\
\hline 5054 & 5051.54 & $16 \mathrm{Fe}$ & 0.0061 & 0.91 & 3.35 \\
\hline 5070 & 5068.79 & $383 \mathrm{Fe}$ & 0.60 & 2.93 & 5.36 \\
\hline 5081 & 5083.34 & $16 \mathrm{Fe}$ & 0.0052 & 0.95 & 3.38 \\
\hline 5110 & 5110.41 & $1 \mathrm{Fe}$ & 0.0014 & 0.00 & 2.41 \\
\hline 5125 & 5123.72 & $16 \mathrm{Fe}$ & & 1.01 & 3.42 \\
\hline 5133 & 5133.68 & $1092 \mathrm{Fe}$ & 13 & 4.16 & 6.56 \\
\hline \multirow[t]{2}{*}{5138} & 5139.26 & $383 \mathrm{Fe}$ & 1.6 & 2.99 & 5.39 \\
\hline & 5139.48 & $383 \mathrm{Fe}$ & 2.0 & 2.93 & 5.33 \\
\hline 5152 & 5153.40 & $8 \mathrm{Na}$ & 0.38 & 2.10 & 4.49 \\
\hline \multirow{6}{*}{5170} & 5166.29 & $1 \mathrm{Fe}$ & & 0.00 & 2.39 \\
\hline & 5167.34 & $2 \mathrm{Mg}$ & 1.2 & 2.70 & 5.09 \\
\hline & 5167.49 & $37 \mathrm{Fe}$ & & 1.55 & 3.91 \\
\hline & 5168.90 & $1 \mathrm{Fe}$ & & 0.05 & 2.44 \\
\hline & 5171.60 & $36 \mathrm{Fe}$ & & 1.48 & 3.86 \\
\hline & 5172.70 & $2 \mathrm{Mg}$ & 3.5 & 2.70 & 5.09 \\
\hline 5184 & 5183.62 & $2 \mathrm{Mg}$ & 6.4 & 2.70 & 5.09 \\
\hline 5207 & 5204.58 & $1 \mathrm{Fe}$ & & 0.09 & 2.46 \\
\hline 5218 & 5216.28 & $36 \mathrm{Fe}$ & 0.064 & 1.60 & 3.97 \\
\hline 5227 & 5227.19 & $37 \mathrm{Fe}$ & 0.27 & 1.55 & 3.91 \\
\hline 5244 & 5241.76 & $36 \mathrm{Fe}$ & & 1.48 & 3.93 \\
\hline 5250 & 5250.65 & $66 \mathrm{Fe}$ & & 2.19 & 4.54 \\
\hline 5261 & 5263.33 & $553 \mathrm{Fe}$ & & 3.25 & 5.60 \\
\hline \multirow[t]{2}{*}{5270} & 5269.54 & $15 \mathrm{Fe}$ & 0.098 & 0.86 & 3.20 \\
\hline & 5270.36 & $37 \mathrm{Fo}$ & 0.20 & 1.60 & 3.94 \\
\hline \multirow[t]{2}{*}{5281} & 5281.80 & $383 \mathrm{Fc}$ & 1.3 & 3.03 & 5.36 \\
\hline & 5283.63 & $553 \mathrm{Fe}$ & & 3.23 & 5.56 \\
\hline 5328 & 5328.05 & $15 \mathrm{Fe}$ & 0.087 & 0.91 & 3.23 \\
\hline \multirow[t]{2}{*}{5340} & 5341.03 & $37 \mathrm{Fe}$ & 0.042 & 1.60 & 3.91 \\
\hline & 5341.06 & $4 \mathrm{Mn}$ & 0.26 & 2.11 & 4.42 \\
\hline 5371 & 5371.49 & $15 \mathrm{Fo}$ & 0.062 & 0.95 & 3.25 \\
\hline
\end{tabular}


TABLE 2.-Wavelength Identifications of Taurid Meteor-Continued

\begin{tabular}{|c|c|c|c|c|c|}
\hline $\begin{array}{c}\lambda \text { mensured } \\
(\AA)\end{array}$ & $\begin{array}{c}\lambda \text { identified } \\
(\AA)\end{array}$ & Multiplet no. & $\begin{array}{c}g A \\
\left(\times 10^{s} / \mathrm{s}\right)\end{array}$ & $\begin{array}{c}E_{1} \\
(\mathrm{eV})\end{array}$ & $\begin{array}{c}E_{2} \\
(\mathrm{eV})\end{array}$ \\
\hline 5397 & 5397.13 & $15 \mathrm{Fe}$ & 0.032 & 0.91 & 3.20 \\
\hline 5403 & 5405.78 & $15 \mathrm{Fe}$ & 0.038 & 0.99 & 3.27 \\
\hline 5423 & 5420.36 & $4 \mathrm{Mn}$ & 0.14 & 2.13 & 4.49 \\
\hline 5429 & 5429.70 & $15 \mathrm{Fc}$ & 0.039 & 0.95 & 3.23 \\
\hline 5445 & 5446.92 & $15 \mathrm{Fe}$ & 0.031 & 0.99 & 3.25 \\
\hline 5454 & 5455.61 & $15 \mathrm{Fe}$ & 0.022 & 1.01 & 3.27 \\
\hline 5472 & 5470.64 & $4 \mathrm{Mn}$ & 0.10 & 2.15 & 4.41 \\
\hline 5497 & 5497.52 & $15 \mathrm{Fe}$ & 0.0084 & 1.01 & 3.25 \\
\hline 5507 & 5506.78 & $15 \mathrm{Fe}$ & 0.0100 & 0.99 & 3.23 \\
\hline 5528 & 5528.46 & $9 \mathrm{Mg}$ & 1.6 & 4.33 & 6.56 \\
\hline 5569 & 5569.62 & $686 \mathrm{Fe}$ & 2.4 & 3.40 & 5.62 \\
\hline 5574 & 5572.85 & $686 \mathrm{Fe}$ & 3.4 & 3.38 & 5.60 \\
\hline 5580 & 5581.97 & $21 \mathrm{Ca}$ & & 2.51 & 4.72 \\
\hline 5585 & 5586.76 & $686 \mathrm{Fe}$ & 4.2 & 3.35 & 5.56 \\
\hline \multirow[t]{2}{*}{5591} & 5588.76 & $21 \mathrm{Ca}$ & & 2.51 & 4.72 \\
\hline & 5594.47 & $21 \mathrm{Ca}$ & & 2.51 & 4.72 \\
\hline \multirow[t]{3}{*}{5602} & 5601.48 & $21 \mathrm{Ca}$ & & 2.51 & 4.72 \\
\hline & 5602.85 & $21 \mathrm{Ca}$ & & 2.51 & 4.72 \\
\hline & 5602.96 & $686 \mathrm{Fe}$ & 0.96 & 3.42 & 5.62 \\
\hline 5614 & 5615.65 & $686 \mathrm{Fe}$ & 4.7 & 3.32 & 5.52 \\
\hline 5660 & 5658.54 & $686 \mathrm{Fe}$ & & 3.38 & 5.56 \\
\hline 5690 & 5688.22 & $6 \mathrm{Na}$ & 1.8 & 2.10 & 4.27 \\
\hline 5710 & 5708.44 & $10 \mathrm{Si}$ & & 4.93 & 7.09 \\
\hline 5772 & 5772.26 & $17 \mathrm{Si}$ & & 5.06 & 7.20 \\
\hline 5790 & 5789.8 & $\mathrm{FeO} \mathrm{B}$ & & & \\
\hline 5798 & 5797.91 & $9 \mathrm{Si}$ & & 4.93 & 7.06 \\
\hline 5859 & 5857.46 & $47 \mathrm{Ca}$ & 3.6 & 2.92 & 5.03 \\
\hline 5891 & 5889.95 & $1 \mathrm{Na}$ & 1.8 & 0.00 & 2.10 \\
\hline 5894 & 5895.92 & $1 \mathrm{Na}$ & 0.90 & 0.00 & 2.09 \\
\hline 5946 & 5948.58 & $16 \mathbf{S i}$ & & 5.06 & 7.14 \\
\hline 6155 & 6154.11 & $5 \mathrm{Na}$ & & 2.10 & 4.10 \\
\hline 6217 & 6218.9 & $\mathrm{FeO} A$ & & & \\
\hline 6347 & 6347.09 & $2 \mathrm{Si} \mathrm{II}$ & & 8.09 & 10.03 \\
\hline 6439 & 6439.07 & $18 \mathrm{Ca}$ & & 2.51 & 4.43 \\
\hline
\end{tabular}

II were computed from the absorption oscillator strengths of Griem (1964). A large number of features in the $3100 \AA$ to $3600 \AA$ region remain unidentified. This spectrum suffers greatly from multiple zero-order star images and poor imagery.

\section{DATA REDUCTION}

The data reduction of the meteor spectrograms consisted of two parts: the wavelength identifications, and the absolute spectral photometry. The method used for the wavelength reductions was to obtain $40 \times$ densitometer tracings of the spectra. Wavelength scales were then con- structed and positioned according to the known wavelengths of strong lines in the speetra. The wavelengths of the meteor radiation were read directly from the constructed scale. These wavelengths were read to the nearest angstrom. This method allows convenient checking of wavelength and relative intensity of lines during identification. Numerous sources were used in checking the wavelengths of the identificd lines. The primary ones were Ceplecha (1964), Halliday (1961, 1969), Harvey (1967a) and Moore (1945). The identifications in the $3100 \AA$ to $3600 \AA$ region were particularly difficult, and many features have remained unidentified. The wavelengths 


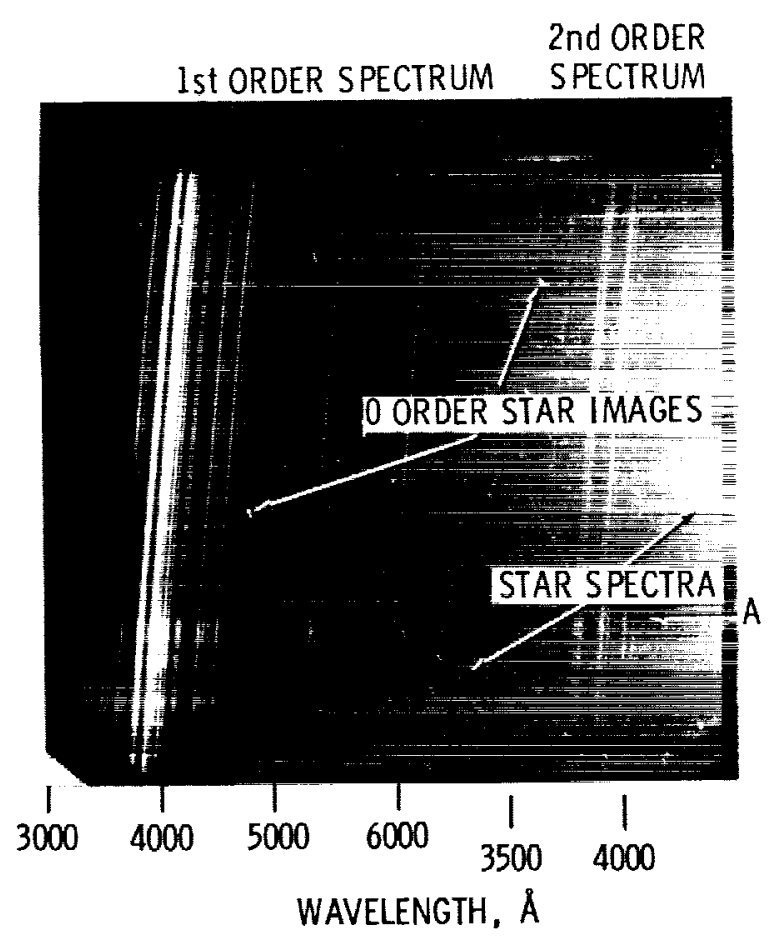

Figure 3.-Enlargement of a spectrogram of a Gemind meteor. and identifications of features in the four spectra are listed in tables 1 to 4 .

The spectral photometry used in the data reduction follows closely that of Harvey (1967b). This photometry is based on calibration of the meteor irrudiance with the irradiance from a quartz-iodine lamp that is a standard of spectral irradiance. Again the $3100 \AA$ to $3600 \AA$ spectral region has proven to be difficult to work in because of the relatively low energy of the standard lamp and the high atmospheric attenuation in this region.

As mentioned in the data section, assumed heights and shower velocities were used for the three shower meteors. Factors which have further degraded the accuracy of the reduced meteor spectral irradiance are: lack of measured atmospheric attenuation, the relativaly unstable character of the film emulsion and developer, poor imagery, and the dense and nonuniform fog background of the spectral plates. The attenuation used here was taken from Elterman (1964).

The reduecd absolute spectral irradiances are

TABLE 3.-Wavelength Identifications of Geminid Meteor

\begin{tabular}{|c|c|c|c|c|c|}
\hline $\begin{array}{c}\lambda \text { measured } \\
(\AA)\end{array}$ & $\begin{array}{c}\lambda \text { identified } \\
(\AA)\end{array}$ & Multiplet no. & $\begin{array}{c}g A \\
\left(\times 10^{\mathrm{B}} / \mathrm{s}\right)\end{array}$ & $\begin{array}{c}E_{1} \\
(\mathrm{eV})\end{array}$ & $\begin{array}{c}E_{2} \\
(\mathrm{eV})\end{array}$ \\
\hline 3363 & 3361.92 & $11 \mathrm{Ca}$ & & 1.89 & 5.56 \\
\hline & 3362.13 & $11 \mathrm{Ca}$ & & 1.89 & 5.56 \\
\hline 3368 & 3369.57 & $6 \mathrm{Ni}$ & 2.1 & 0.00 & 3.66 \\
\hline 3371 & 3370.79 & $304 \mathrm{Fe}$ & 32 & 2.68 & 6.34 \\
\hline & 3371.99 & $7 \mathrm{Ni}$ & 0.41 & 0.16 & 2.84 \\
\hline 3378 & 3379.02 & $85 \mathrm{Fe}$ & & 2.17 & 5.82 \\
\hline 3388 & 3388.17 & $23 \mathrm{Co}$ & 2.2 & 0.58 & 4.22 \\
\hline 3400 & 3399.34 & $85 \mathrm{Fe}$ & 25 & 2.19 & 5.82 \\
\hline & 3401.52 & $26 \mathrm{Fe}$ & & 0.91 & 4.54 \\
\hline 3407 & 3405.12 & $23 \mathrm{Co}$ & 15 & 0.43 & 4.05 \\
\hline & 3407.46 & $83 \mathrm{Fe}$ & 33 & 2.17 & 5.79 \\
\hline 3408 & 3409.18 & $23 \mathrm{Co}$ & 7.3 & 0.51 & 4.13 \\
\hline 3412 & 3413.14 & $85 \mathrm{Fe}$ & 26 & 2.19 & 5.80 \\
\hline 3416 & 3414.76 & $19 \mathrm{Ni}$ & 5.7 & 0.03 & 3.64 \\
\hline & 3417.84 & $81 \mathrm{Fe}$ & 18 & 2.21 & 5.82 \\
\hline 3420 & 3418.51 & $81 \mathrm{Fe}$ & 18 & 2.21 & 5.82 \\
\hline & 3422.66 & $85 \mathrm{Fe}$ & 9.3 & 2.21 & 5.82 \\
\hline 3425 & 3424.29 & $81 \mathrm{Fe}$ & 17 & 2.17 & 5.77 \\
\hline 3441 & 3440.61 & $6 \mathrm{Fe}$ & 2.8 & 0.00 & 3.59 \\
\hline & 3440.99 & $6 \mathrm{Fc}$ & 0.64 & 0.05 & 3.64 \\
\hline 3449 & 3450.33 & $82 \mathrm{Fc}$ & 8.9 & 2.21 & 5.79 \\
\hline 3459 & 3458.47 & $19 \mathrm{Ni}$ & 4.9 & 0.21 & 3.78 \\
\hline
\end{tabular}


TABle 3.-Wavelength Identifications of Geminid Meteor-Continued

\begin{tabular}{|c|c|c|c|c|c|}
\hline $\begin{array}{c}\lambda \text { measured } \\
(\AA ̊)\end{array}$ & $\begin{array}{c}\lambda \text { identified } \\
(\AA)\end{array}$ & Multiplet no. & $\begin{array}{c}g A \\
\left(\times 10^{8} / \mathrm{s}\right)\end{array}$ & $\begin{array}{c}E_{1} \\
(\mathrm{eV})\end{array}$ & $\begin{array}{c}E_{2} \\
(\mathrm{eV})\end{array}$ \\
\hline \multirow[t]{2}{*}{3462} & 3461.65 & $17 \mathrm{Ni}$ & 3.2 & 0.03 & 3.59 \\
\hline & 3462.80 & $23 \mathrm{Co}$ & 9.7 & 0.63 & 4.19 \\
\hline 3466 & 3465.86 & $6 \mathrm{Fe}$ & 0.52 & 0.11 & 3.67 \\
\hline 3472 & 3472.54 & $20 \mathrm{Ni}$ & 1.2 & 0.11 & 3.66 \\
\hline \multirow[t]{2}{*}{3474} & 3474.02 & $4 \mathrm{Co}$ & 3.6 & 0.00 & 3.55 \\
\hline & 3475.45 & $6 \mathrm{Fe}$ & 0.64 & 0.09 & 3.64 \\
\hline 3485 & 3485.34 & $78 \mathrm{Fe}$ & 5.9 & 2.19 & 5.73 \\
\hline 3490 & 3490.58 & $6 \mathrm{Fe}$ & 0.58 & 0.05 & 3.59 \\
\hline 3495 & 3495.29 & $238 \mathrm{Fe}$ & 11 & 2.55 & 6.08 \\
\hline \multirow[t]{2}{*}{3497} & 3497.11 & $78 \mathrm{Fe}$ & 7.4 & 2.19 & 5.70 \\
\hline & 3497.84 & $6 \mathrm{Fe}$ & 0.19 & 0.11 & 3.64 \\
\hline \multirow[t]{2}{*}{3499} & 3500.57 & $238 \mathrm{Fe}$ & & 2.58 & 6.10 \\
\hline & 3500.85 & $6 \mathrm{Ni}$ & & 0.16 & 3.69 \\
\hline 3514 & 3513.82 & $24 \mathrm{Fe}$ & 1.7 & 0.86 & 4.37 \\
\hline 3521 & 3521.26 & $24 \mathrm{Fe}$ & 1.7 & 0.91 & 4.42 \\
\hline 3524 & 3524.54 & $18 \mathrm{Ni}$ & 4.6 & 0.03 & 3.53 \\
\hline \multirow[t]{2}{*}{3527} & 3526.04 & $6 \mathrm{Fe}$ & 0.13 & 0.09 & 3.59 \\
\hline & 3526.17 & $24 \mathrm{Fe}$ & 0.69 & 0.95 & 4.45 \\
\hline 3531 & 3533.20 & $326 \mathrm{Fe}$ & 23 & 2.87 & 6.36 \\
\hline 3536 & 3536.56 & $326 \mathrm{Fe}$ & 56 & 2.86 & 6.35 \\
\hline \multirow[t]{2}{*}{3542} & 3541.09 & $326 \mathrm{Fe}$ & 65 & 2.84 & 6.32 \\
\hline & 3542.08 & $326 \mathrm{Fe}$ & 61 & 2.85 & 6.34 \\
\hline 3566 & 3565.38 & $24 \mathrm{Fe}$ & 7.8 & 0.95 & 4.42 \\
\hline \multirow[t]{2}{*}{3570} & 3570.10 & $24 \mathrm{Fe}$ & 18 & 0.91 & 4.37 \\
\hline & 3570.24 & $326 \mathrm{Fe}$ & & 2.80 & 6.25 \\
\hline 3581 & 3581.20 & $23 \mathrm{Fe}$ & 23 & 0.86 & 4.30 \\
\hline 3596 & 3594.64 & $322 \mathrm{Fe}$ & 21 & 2.84 & 6.27 \\
\hline \multirow[t]{2}{*}{3606} & 3605.46 & $294 \mathrm{Fe}$ & 51 & 2.72 & 6.14 \\
\hline & 3606.68 & $294 \mathrm{Fe}$ & 65 & 2.68 & 6.10 \\
\hline 3608 & 3608.86 & $23 \mathrm{Fe}$ & 10 & 1.01 & 4.43 \\
\hline 3618 & 3618.77 & $23 \mathrm{Fe}$ & 9.5 & 0.99 & 4.40 \\
\hline 3621 & 3621.46 & $294 \mathrm{Fe}$ & 50 & 2.72 & 6.12 \\
\hline 3631 & 3631.46 & $23 \mathrm{Fe}$ & 8.6 & 0.95 & 4.35 \\
\hline \multirow[t]{2}{*}{3640} & 3638.30 & $294 \mathrm{Fe}$ & 28 & 2.75 & 6.14 \\
\hline & 3640.39 & $295 \mathrm{Fe}$ & 45 & 2.72 & 6.11 \\
\hline 3644 & 3644.41 & $9 \mathrm{Ca}$ & 1.8 & 1.89 & 5.28 \\
\hline \multirow[t]{2}{*}{3649} & 3647.84 & $24 \mathrm{Fe}$ & 6.1 & 0.91 & 4.29 \\
\hline & 3649.30 & $5 \mathrm{Fe}$ & & 0.00 & 3.38 \\
\hline 3660 & 3659.52 & $180 \mathrm{Fe}$ & 9.9 & 2.44 & 5.82 \\
\hline 3671 & 3669.52 & $291 \mathrm{Fe}$ & 32 & 2.72 & 6.08 \\
\hline 3680 & 3679.92 & $5 \mathrm{Fe}$ & 0.29 & 0.00 & 3.35 \\
\hline \multirow[t]{2}{*}{3685} & 3684.11 & $292 \mathrm{Fe}$ & 21 & 2.72 & 6.07 \\
\hline & 3686.00 & $385 \mathrm{Fe}$ & 34 & 2.93 & 6.28 \\
\hline 3687 & 3687.46 & $21 \mathrm{Fe}$ & 2.5 & 0.86 & 4.20 \\
\hline \multirow[t]{2}{*}{3696} & 3694.01 & $394 \mathrm{Fe}$ & 72 & 3.03 & 6.37 \\
\hline & 3695.05 & $229 \mathrm{Fe}$ & 12 & 2.58 & 5.92 \\
\hline 3698 & 3697.43 & $389 \mathrm{Fe}$ & & 2.99 & 6.32 \\
\hline \multirow[t]{2}{*}{3707} & 3705.57 & $5 \mathrm{Fe}$ & 0.38 & 0.05 & 3.38 \\
\hline & 3707.82 & $5 \mathrm{Fe}$ & 0.14 & 0.09 & 3.42 \\
\hline \multirow[t]{2}{*}{3720} & 3719.94 & $5 \mathrm{Fe}$ & 2.5 & 0.00 & 3.32 \\
\hline & 3722.56 & $5 \mathrm{Fe}$ & 0.40 & 0.09 & 3.40 \\
\hline \multirow[t]{2}{*}{3738} & 3733.40 & $5 \mathrm{Fe}$ & 0.36 & 0.11 & 3.42 \\
\hline & 3734.87 & $21 \mathrm{Fe}$ & 20 & 0.86 & 4.16 \\
\hline
\end{tabular}


TABLF 3.-Wavelength Identifications of Geminid Meteor-Continued

\begin{tabular}{|c|c|c|c|c|c|}
\hline $\begin{array}{c}\lambda \text { measured } \\
(\AA)\end{array}$ & $\begin{array}{c}\lambda \text { identified } \\
(\AA)\end{array}$ & Multiplet no. & $\begin{array}{c}0 A \\
\left(\times 10^{8} / \mathrm{s}\right)\end{array}$ & $\begin{array}{c}E_{1} \\
(\mathrm{eV})\end{array}$ & $\begin{array}{c}E_{2} \\
(\mathrm{eV})\end{array}$ \\
\hline \multirow{5}{*}{3747} & 3737.13 & $5 \mathrm{Fe}$ & 1.5 & 0.05 & 3.35 \\
\hline & 3745.56 & $5 \mathrm{Fe}$ & 1.2 & 0.09 & 3.38 \\
\hline & 3745.90 & $5 \mathrm{Fe}$ & 0.31 & 0.12 & 3.42 \\
\hline & 3748.26 & $5 \mathrm{Fe}$ & 0.71 & 0.11 & 3.40 \\
\hline & 3749.49 & $20 \mathrm{Fe}$ & 13 & 0.91 & 4.20 \\
\hline 3759 & 3758.24 & $21 \mathrm{Fe}$ & 10 & 0.95 & 4.24 \\
\hline 3763 & 3763.79 & $21 \mathrm{Fe}$ & 6.2 & 0.99 & 4.26 \\
\hline 3788 & 3787.88 & $21 \mathrm{Fe}$ & 1.7 & 1.01 & 4.26 \\
\hline 3794 & 3795.00 & $22 \mathrm{Fe}$ & 2.3 & 0.99 & 4.24 \\
\hline \multirow[t]{2}{*}{3798} & 3798.51 & $21 \mathrm{Fe}$ & 0.93 & 0.91 & 4.16 \\
\hline & 3799.55 & $21 \mathrm{Fe}$ & 1.5 & 0.95 & 4.20 \\
\hline \multirow[t]{2}{*}{3806} & 3805.34 & $608 \mathrm{Fe}$ & 45 & 3.29 & 6.53 \\
\hline & 3806.70 & $607 \mathrm{Fe}$ & 21 & 3.25 & 6.50 \\
\hline 3815 & 3815.84 & $45 \mathrm{Fe}$ & 16 & 1.48 & 4.71 \\
\hline \multirow[t]{2}{*}{3830} & 3827.82 & $45 \mathrm{Fe}$ & 15 & 1.55 & 4.77 \\
\hline & 3829.35 & $3 \mathrm{Mg}$ & 11 & 2.70 & 5.92 \\
\hline 3833 & 3832.51 & $3 \mathrm{Mg}$ & 23 & 2.70 & 5.92 \\
\hline 3838 & 3838.26 & $3 \mathrm{Mg}$ & 39 & 2.70 & 5.92 \\
\hline 3847 & 3846.80 & $664 \mathrm{Fe}$ & 20 & 3.24 & 6.45 \\
\hline 3860 & 3859.91 & $4 \mathrm{Fe}$ & 1.4 & 0.00 & 3.20 \\
\hline 3871 & 3872.50 & $20 \mathrm{Fe}$ & 1.0 & 0.99 & 4.17 \\
\hline \multirow[t]{2}{*}{3879} & 3878.02 & $20 \mathrm{Fe}$ & 1.4 & 0.95 & 4.14 \\
\hline & 3878.58 & $4 \mathrm{Fe}$ & 0.33 & 0.09 & 3.27 \\
\hline \multirow[t]{2}{*}{3887} & 3886.28 & $4 \mathrm{Fe}$ & 0.63 & 0.05 & 3.23 \\
\hline & 3887.05 & $20 \mathrm{Fe}$ & 4.2 & 0.91 & 4.09 \\
\hline 3896 & 3895.66 & $4 \mathrm{Fe}$ & 0.14 & 0.11 & 3.28 \\
\hline 3900 & 3899.71 & $4 \mathrm{Fe}$ & 0.21 & 0.09 & 3.25 \\
\hline \multirow[t]{2}{*}{3905} & 3905.53 & $3 \mathrm{Si}$ & 0.86 & 1.90 & 5.06 \\
\hline & 3906.48 & $4 \mathrm{Fe}$ & 0.055 & 0.11 & 3.27 \\
\hline 3924 & 3922.91 & $4 \mathrm{Fe}$ & 0.18 & 0.05 & 3.20 \\
\hline \multirow[t]{2}{*}{3935} & 3930.30 & $4 \mathrm{Fe}$ & 0.27 & 0.09 & 3.23 \\
\hline & 3933.67 & $1 \mathrm{Ca}$ II & 0.91 & 0.00 & 3.14 \\
\hline 3945 & 3944.03 & $1 \mathrm{AI}$ & 0.66 & 0.00 & 3.13 \\
\hline 3948 & 3948.78 & $604 \mathrm{Fe}$ & 11 & 3.25 & 6.38 \\
\hline 3962 & 3961.53 & $1 \mathrm{AI}$ & 1.3 & 0.00 & 3.13 \\
\hline \multirow[t]{2}{*}{3969} & 3968.47 & $1 \mathrm{Ca}$ II & 0.45 & 0.00 & 3.11 \\
\hline & 3969.26 & $43 \mathrm{Fe}$ & 4.4 & 1.48 & 4.59 \\
\hline 3983 & 3983.96 & $277 \mathrm{Fe}$ & 5.4 & 2.72 & 5.81 \\
\hline \multirow[t]{2}{*}{3999} & 3997.40 & $278 \mathrm{Fe}$ & 11 & 2.72 & 5.80 \\
\hline & 3998.06 & $276 \mathrm{Fe}$ & 3.7 & 2.68 & 5.77 \\
\hline 4005 & 4005.25 & $43 \mathrm{Fe}$ & 3.6 & 1.55 & 4.63 \\
\hline 4008 & 4007.27 & $277 \mathrm{Fe}$ & & 2.75 & 5.83 \\
\hline 4011 & 4009.72 & $72 \mathrm{Fe}$ & 1.4 & 2.21 & 5.29 \\
\hline 4032 & 4030.76 & $2 \mathrm{Mn}$ & 1.4 & 0.00 & 3.06 \\
\hline \multirow[t]{2}{*}{4035} & 4033.07 & $2 \mathrm{Mn}$ & 0.95 & 0.00 & 3.06 \\
\hline & 4034.49 & $2 \mathrm{Mn}$ & 0.54 & 0.00 & 3.06 \\
\hline 4046 & 4045.82 & $43 \mathrm{Fe}$ & 22 & 1.48 & 4.53 \\
\hline 4063 & 4063.60 & $43 \mathrm{Fe}$ & 9.9 & 1,55 & 4.59 \\
\hline 4069 & 4067.98 & $559 \mathrm{Fe}$ & & 3.20 & 6.23 \\
\hline 4072 & 4071.74 & $43 \mathrm{Fe}$ & 9.1 & 1.60 & 4.63 \\
\hline 4101 & 4100.74 & $18 \mathrm{Fe}$ & & 0.86 & 3.86 \\
\hline 4109 & 4107.49 & $354 \mathrm{Fe}$ & 5.6 & 2.82 & 5.82 \\
\hline 4115 & 4114.45 & $357 \mathrm{Fe}$ & & 2.82 & 5.82 \\
\hline
\end{tabular}


TABle 3.-Wavelength Identifications of Geminid Meteor-Continued

\begin{tabular}{|c|c|c|c|c|c|}
\hline $\begin{array}{c}\lambda \text { measured } \\
(\AA)\end{array}$ & $\begin{array}{c}\lambda \text { identified } \\
(\AA)\end{array}$ & Multiplet no. & $\begin{array}{c}g A \\
\left(\times 10^{8} / \mathrm{s}\right)\end{array}$ & $\begin{array}{c}E_{1} \\
(\mathrm{eV})\end{array}$ & $\begin{array}{c}E_{2} \\
(\mathrm{eV})\end{array}$ \\
\hline 4119 & 4118.55 & $801 \mathrm{Fe}$ & 33 & 3.56 & 6.55 \\
\hline \multirow[t]{2}{*}{4122} & 4121.32 & $28 \mathrm{Co}$ & 3.7 & 0.92 & 3.91 \\
\hline & 4121.81 & $356 \mathrm{Fe}$ & & 2.82 & 5.81 \\
\hline 4133 & 4132.06 & $43 \mathrm{Fe}$ & 2.7 & 1.60 & 4.59 \\
\hline 4136 & 4134.68 & $357 \mathrm{Fe}$ & 5.5 & 2.82 & 5.80 \\
\hline 4144 & 4143.87 & $43 \mathrm{Fe}$ & 2.9 & 1.55 & 4.53 \\
\hline 4151 & 4152.17 & $18 \mathrm{Fe}$ & & 0.95 & 3.93 \\
\hline \multirow[t]{2}{*}{4153} & 4154.50 & $355 \mathrm{Fe}$ & 5.3 & 2.82 & 5.79 \\
\hline & 4154.81 & $694 \mathrm{Fe}$ & & 3.35 & 6.32 \\
\hline 4171 & 4172.75 & $19 \mathrm{Fe}$ & & 0.95 & 3.91 \\
\hline 4176 & 4175.64 & $354 \mathrm{Fe}$ & 4.7 & 2.83 & 5.79 \\
\hline 4181 & 4181.76 & $354 \mathrm{Fe}$ & 10 & 2.82 & 5.77 \\
\hline 4184 & 4184.90 & $355 \mathrm{Fe}$ & 3.9 & 2.82 & 5.77 \\
\hline \multirow[t]{3}{*}{4190} & 4187.04 & $152 \mathrm{Fe}$ & 6.9 & 2.44 & 5.39 \\
\hline & 4187.80 & $152 \mathrm{Fe}$ & 6.5 & 2.41 & 5.36 \\
\hline & 4191.44 & $152 \mathrm{Fe}$ & 4.4 & 2.46 & 5.40 \\
\hline 4200 & 4199.10 & $522 \mathrm{Fe}$ & 25 & 3.03 & 5.97 \\
\hline 4202 & 4202.03 & $42 \mathrm{Fe}$ & 2.0 & 1.48 & 4.42 \\
\hline 4209 & 4210.35 & $152 \mathrm{Fe}$ & 2.2 & 2.47 & 5.40 \\
\hline \multirow[t]{2}{*}{4218} & 4216.19 & $3 \mathrm{Fe}$ & 0.0031 & 0.00 & 2.93 \\
\hline & 4219.36 & $800 \mathrm{Fe}$ & 27 & 3.56 & 6.48 \\
\hline \multirow[t]{2}{*}{4227} & 4226.73 & $2 \mathrm{Ca}$ & 1 & 0.00 & 2.92 \\
\hline & 4227.43 & $693 \mathrm{Fc}$ & 38 & 3.32 & 6.24 \\
\hline 4234 & 4233.61 & $152 \mathrm{Fe}$ & 5.9 & 2.47 & 5.39 \\
\hline 4237 & 4235.94 & $152 \mathrm{Fe}$ & 7.9 & 2.41 & 5.33 \\
\hline \multirow[t]{2}{*}{4252} & 4250.79 & $42 \mathrm{Fe}$ & 1.5 & 1.55 & 4.45 \\
\hline & 4250.13 & $152 \mathrm{Fe}$ & & 2.46 & 5.36 \\
\hline 4254 & 4254.35 & $1 \mathrm{Cr}$ & 2.0 & 0.00 & 2.90 \\
\hline 4260 & 4260.48 & $152 \mathrm{Fe}$ & 15 & 2.39 & 5.29 \\
\hline 4272 & 4271.76 & $42 \mathrm{Fe}$ & 5.2 & 1.48 & 4.37 \\
\hline 4282 & 4282.41 & $71 \mathrm{Fe}$ & 2.0 & 2.17 & 5.05 \\
\hline \multirow[t]{2}{*}{4291} & 4289.72 & $1 \mathrm{Cr}$ & 0.95 & 0.00 & 2.88 \\
\hline & 4291.66 & $3 \mathrm{Fe}$ & & 0.09 & 2.99 \\
\hline 4294 & 4294.13 & $41 \mathrm{Fe}$ & 0.71 & 1.48 & 4.35 \\
\hline 4300 & 4299.24 & $152 \mathrm{Fe}$ & 5.2 & 2.41 & 5.29 \\
\hline 4302 & 4302.53 & $5 \mathrm{Ca}$ & 7.1 & 1.89 & 4.76 \\
\hline 4308 & 4307.91 & $42 \mathrm{Fe}$ & 5.9 & 1.55 & 4.42 \\
\hline 4315 & 4315.09 & $71 \mathrm{Fe}$ & 1.5 & 2.19 & 5.05 \\
\hline 4319 & 4318.65 & $5 \mathrm{Ca}$ & 2.5 & 1.89 & 4.75 \\
\hline 4326 & 4325.76 & $42 \mathrm{Fc}$ & 6.1 & 1.60 & 4.45 \\
\hline 4336 & 4337.05 & $41 \mathrm{Fe}$ & 0.23 & 1.55 & 4.40 \\
\hline \multirow[t]{2}{*}{4339} & 4339.45 & $22 \mathrm{Cr}$ & 0.93 & 0.98 & 3.82 \\
\hline & 4339.72 & $22 \mathrm{Cr}$ & 0.30 & 0.96 & 3.80 \\
\hline 4351 & 4351.77 & $22 \mathrm{Cr}$ & 2.0 & 1.03 & 3.86 \\
\hline 4353 & 4352.74 & $71 \mathrm{Fe}$ & 1.0 & 2.21 & 5.05 \\
\hline 4358 & 4358.51 & $412 \mathrm{Fe}$ & & 2.94 & 5.77 \\
\hline 4377 & 4375.93 & $2 \mathrm{Fe}$ & 0.0094 & 0.00 & 2.82 \\
\hline 4384 & 4383.55 & $41 \mathrm{Fe}$ & 7.7 & 1.48 & 4.29 \\
\hline 4404 & 4404.75 & $41 \mathrm{Fe}$ & 4.4 & 1.55 & 4.35 \\
\hline 4415 & 4415.12 & $41 \mathrm{Fe}$ & 2.8 & 1.60 & 4.40 \\
\hline 4426 & 4427.31 & $2 \mathrm{Fe}$ & 0.0099 & 0.05 & 2.84 \\
\hline \multirow[t]{2}{*}{4434} & 4434.96 & $4 \mathrm{Ca}$ & 3.5 & 1.88 & 4.66 \\
\hline & 4435.69 & $4 \mathrm{Ca}$ & 0.96 & 1.88 & 4.66 \\
\hline
\end{tabular}


TABIr: 3,-Wavelength Identifications of Geminid Meteor-Continued

\begin{tabular}{|c|c|c|c|c|c|}
\hline $\begin{array}{l}\lambda \text { measured } \\
(\AA)\end{array}$ & $\begin{array}{c}\lambda \text { identified } \\
(\AA)\end{array}$ & Multiplet no. & $\begin{array}{c}g A \\
\left(\times 10^{8} / s\right)\end{array}$ & $\begin{array}{c}E_{1} \\
(\mathrm{eV})\end{array}$ & $\begin{array}{c}E_{2} \\
(\mathrm{eV})\end{array}$ \\
\hline 4442 & 4442.34 & $68 \mathrm{Fe}$ & & 2.19 & 4.97 \\
\hline \multirow[t]{2}{*}{4455} & 4454.78 & $4 \mathrm{Ca}$ & 7.5 & 1.89 & 4.66 \\
\hline & 4455.89 & $4 \mathrm{Ca}$ & 0.97 & 1.89 & 4.66 \\
\hline 4462 & 4461.65 & $2 \mathrm{Fe}$ & 0.0052 & 0.09 & 2.85 \\
\hline 4467 & 4466.55 & $350 \mathrm{Fe}$ & 5.3 & 2.82 & 5.58 \\
\hline 4482 & 4482.17 & $2 \mathrm{Fe}$ & 0.0053 & 0.11 & 2.86 \\
\hline 4531 & 4531.15 & $39 \mathrm{Fe}$ & 0.076 & 1.48 & 4.20 \\
\hline 4580 & 4581.40 & $23 \mathrm{Ca}$ & 0.96 & 2.51 & 5.21 \\
\hline 4585 & 4585.87 & $23 \mathrm{Ca}$ & 1.5 & 2.51 & 5.21 \\
\hline 4646 & 4647.44 & $409 \mathrm{Fe}$ & & 2.94 & 5.59 \\
\hline 4878 & 4878.22 & $318 \mathrm{Fe}$ & 0.77 & 2.87 & 5.40 \\
\hline \multirow[t]{2}{*}{4890} & 4890.77 & $318 \mathrm{Fc}$ & 2.2 & 2.86 & 5.39 \\
\hline & 4891.50 & $318 \mathrm{Fe}$ & 4.7 & 2.84 & 5.36 \\
\hline \multirow[t]{2}{*}{4920} & 4919.00 & $318 \mathrm{Fe}$ & 2.9 & 2.85 & 5.38 \\
\hline & 4920.50 & $318 \mathrm{Fe}$ & 6.5 & 2.82 & 5.33 \\
\hline \multirow[t]{2}{*}{4960} & 4957.31 & $318 \mathrm{Fe}$ & 3.2 & 2.84 & 5.33 \\
\hline & 4957.61 & $318 \mathrm{Fe}$ & 6.4 & 2.80 & 5.29 \\
\hline 5007 & 5006.13 & $318 \mathrm{Fe}$ & 1.3 & 2.82 & 5.29 \\
\hline 5108 & 5110.41 & $1 \mathrm{Fe}$ & 0.0014 & 0.00 & 2.41 \\
\hline \multirow[t]{4}{*}{5167} & 5166.29 & $1 \mathrm{Fe}$ & & 0.00 & 2.41 \\
\hline & 5167.34 & $2 \mathrm{Mg}_{\mathrm{g}}$ & 1.2 & 2.70 & 5.09 \\
\hline & 5167.49 & $37 \mathrm{Fe}$ & 0.26 & 1.48 & 3.87 \\
\hline & 5168.90 & $1 \mathrm{Fe}$ & & 0.05 & 2.44 \\
\hline \multirow[t]{2}{*}{5173} & 5171.60 & $36 \mathrm{Fe}$ & 0.12 & 1.48 & 3.86 \\
\hline & 5172.70 & $2 \mathrm{Mg}$ & 3.5 & 2.70 & 5.09 \\
\hline 5184 & 5183.62 & $2 \mathrm{Mg}$ & 6.4 & 2.70 & 5.09 \\
\hline \multirow[t]{2}{*}{5270} & 5269.54 & $15 \mathrm{Fe}$ & 0.098 & 0.86 & 3.20 \\
\hline & 5270.36 & $37 \mathrm{Fe}$ & 0.20 & 1.60 & 3.94 \\
\hline 5329 & 5328.05 & $15 \mathrm{Fc}$ & 0.087 & 0.91 & 3.23 \\
\hline \multirow[t]{2}{*}{5893} & 5889.95 & $1 \mathrm{Na}$ & 1.8 & 0.00 & 2.10 \\
\hline & 5895.92 & $1 \mathrm{Na}$ & 0.9 & 0.00 & 2.09 \\
\hline
\end{tabular}

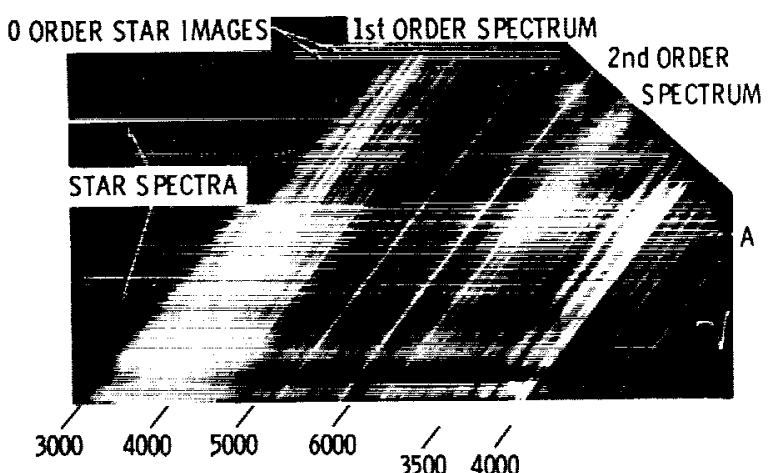

WAVELENGTH, A

Figure 4.--Enlargement of a spectrogram of a Perseid meteor. probably within a factor of 2 of the actual values, except perhaps for those in the near-ultraviolet region. In many cases, rolative moasurement of two lines in a spectrum (upon which most of the analysis is based) is estimated to be of the order of 5 to 10 pereent accuracy. However, in some cases, espocially whore imagery is poor, the relative photometry suffers from blending of lines and the relative measurements aro loss accurate. The spectral irradiances of the four meteors are shown in figures 5 to 8 .

\section{DATA ANALYSIS}

The data analysis is based on the assumption that the population distribution of the excited states is a Boltzmann distribution. Agrecment in temperatures calculated from pairs of lines from 
TABLE 4.-Wavelength Identifications of Perseid Meleor

\begin{tabular}{|c|c|c|c|c|c|}
\hline $\begin{array}{c}\lambda \text { measured } \\
(\AA)\end{array}$ & $\begin{array}{c}\lambda \text { identified } \\
\text { (§) }\end{array}$ & Multiplet no. & $\begin{array}{c}g A \\
\left(\times 10^{8} / \mathrm{s}\right)\end{array}$ & $\begin{array}{c}E_{\mathrm{I}} \\
(\mathrm{eV})\end{array}$ & $\begin{array}{c}E_{2} \\
(\mathrm{eV})\end{array}$ \\
\hline 3090 & 3091.58 & $28 \mathrm{Fe}$ & 3.2 & 1.01 & 5.00 \\
\hline \multirow[t]{4}{*}{3102} & 3099.97 & $28 \mathrm{Fe}$ & 8.6 & 0.91 & 4.89 \\
\hline & 3100.30 & $28 \mathrm{Fe}$ & 3.9 & 0.99 & 4.97 \\
\hline & 3100.67 & $28 \mathrm{Fe}$ & 3.6 & 0.95 & 4.93 \\
\hline & 3101.55 & $25 \mathrm{Ni}$ & 4.6 & 0.11 & 4.09 \\
\hline 3135 & 3134.11 & $25 \mathrm{Ni}$ & 5.8 & 0.21 & 4.15 \\
\hline \multirow[t]{2}{*}{3192} & 3191.66 & $8 \mathrm{Fe}$ & 0.087 & 0.00 & 3.87 \\
\hline & 3193.21 & $7 \mathrm{Fe}$ & 0.14 & 0.00 & 3.86 \\
\hline 3240 & 3239.44 & $157 \mathrm{Fe}$ & 65 & 2.41 & 6.22 \\
\hline 3265 & 3265.62 & $91 \mathrm{Fe}$ & 18 & 2.17 & 5.95 \\
\hline \multirow[t]{2}{*}{3298} & 3302.32 & $2 \mathrm{Na}$ & 0.65 & 0.00 & 3.74 \\
\hline & 3302.99 & $2 \mathrm{Na}$ & 0.33 & 0.00 & 3.74 \\
\hline \multirow[t]{3}{*}{3348} & 3344.51 & $11 \mathrm{Ca}$ & & 1.87 & 5.56 \\
\hline & 3350.21 & $11 \mathrm{Ca}$ & & 1.88 & 5.56 \\
\hline & 3350.36 & $11 \mathrm{Ca}$ & & 1.88 & 5.56 \\
\hline 3370 & 3369.57 & $6 \mathrm{Ni}$ & 2.1 & 0.00 & 3.66 \\
\hline \multirow{2}{*}{3385} & 3383.69 & $85 \mathrm{Fe}$ & & 2.19 & 5.84 \\
\hline & 3383.98 & $83 \mathrm{Fe}$ & 9.1 & 2.17 & 5.81 \\
\hline \multirow[t]{3}{*}{3422} & 3418.51 & $81 \mathrm{Fe}$ & 18 & 2.21 & 5.82 \\
\hline & 3422.66 & $85 \mathrm{Fe}$ & 9.3 & 2.21 & 5.82 \\
\hline & 3424.29 & $81 \mathrm{Fe}$ & 17 & 2.17 & 5.77 \\
\hline \multirow[t]{2}{*}{3441} & 3440.61 & $6 \mathrm{Fe}$ & 2.8 & 0.00 & 3.59 \\
\hline & 3440.99 & $6 \mathrm{Fe}$ & 0.64 & 0.05 & 3.64 \\
\hline \multirow[t]{2}{*}{3472} & 3474.02 & $4 \mathrm{Co}$ & 3.6 & 0.11 & 3.66 \\
\hline & 3475.45 & $6 \mathrm{Fe}$ & 0.64 & 0.09 & 3.64 \\
\hline 3487 & 3485.34 & $78 \mathrm{Fe}$ & 5.9 & 2.19 & 5.73 \\
\hline 3495 & 3495.29 & $238 \mathrm{Fe}$ & 11 & 2.55 & 6.08 \\
\hline \multirow{2}{*}{3566} & 3565.38 & $24 \mathrm{Fe}$ & 7.8 & 0.95 & 4.42 \\
\hline & 3570.10 & $24 \mathrm{Fe}$ & 18 & 0.91 & 4.37 \\
\hline 3581 & 3581.20 & $23 \mathrm{Fe}$ & 23 & 0.86 & 4.30 \\
\hline 3608 & 3608.86 & $23 \mathrm{Fe}$ & 10 & 1.01 & 4.43 \\
\hline 3618 & 3618.77 & $23 \mathrm{Fe}$ & 9.5 & 0.99 & 4.40 \\
\hline 3630 & 3631.46 & $23 \mathrm{Fe}$ & 8.6 & 0.95 & 4.35 \\
\hline 3649 & 3647.84 & $24 \mathrm{Fe}$ & 6.1 & 0.91 & 4.29 \\
\hline \multirow[t]{2}{*}{3685} & 3684.11 & $292 \mathrm{Fe}$ & 21 & 2.72 & 6.07 \\
\hline & 3686.00 & $385 \mathrm{Fe}$ & 34 & 2.93 & 6.28 \\
\hline \multirow{2}{*}{3706} & 3705.57 & $5 \mathrm{Fe}$ & 0.38 & 0.05 & 3.38 \\
\hline & 3707.82 & $5 \mathrm{Fe}$ & 0.41 & 0.09 & 3.41 \\
\hline \multirow[t]{2}{*}{3720} & 3719.94 & $5 \mathrm{Fe}$ & 2.5 & 0.00 & 3.32 \\
\hline & 3722.56 & $5 \mathrm{Fe}$ & 0.40 & 0.09 & 3.40 \\
\hline \multirow[t]{3}{*}{3735} & 3733.40 & $5 \mathrm{Fe}$ & 0.36 & 0.11 & 3,42 \\
\hline & 3734.87 & $21 \mathrm{Fe}$ & 20 & 0.86 & 4.16 \\
\hline & 3737.13 & $5 \mathrm{Fe}$ & 1.5 & 0.05 & 3.35 \\
\hline \multirow[t]{4}{*}{3746} & 3745.56 & $5 \mathrm{Fe}$ & 1.2 & 0.09 & 3.38 \\
\hline & 3745.90 & $5 \mathrm{Fe}$ & 0.31 & 0.12 & 3.42 \\
\hline & 3748.26 & $5 \mathrm{Fe}$ & 0.71 & 0.11 & 3.40 \\
\hline & 3749.49 & $20 \mathrm{Fe}$ & 13 & 0.91 & 4.20 \\
\hline 3760 & 3758.24 & $21 \mathrm{Fe}$ & 10 & 0.95 & 4.24 \\
\hline \multirow{5}{*}{$\begin{array}{l}3796 \\
3836\end{array}$} & 3795.00 & $22 \mathrm{Fe}$ & 2.3 & 0.99 & 4.24 \\
\hline & 3827.82 & $45 \mathrm{Fe}$ & 15 & 1.55 & 4.77 \\
\hline & 3829.35 & $3 \mathrm{Mg}$ & 11 & 2.70 & 5.92 \\
\hline & 3832.51 & $3 \mathrm{Mg}$ & 23 & 2.70 & 5.92 \\
\hline & 3838.26 & $3 \mathrm{Mg}$ & 39 & 2.70 & 5.92 \\
\hline 3860 & 3859.91 & $4 \mathrm{Fe}$ & 1.4 & 0.00 & 3.20 \\
\hline
\end{tabular}


TARIE 4.-Wavelength Identifications of Perseid Meteor-Continued

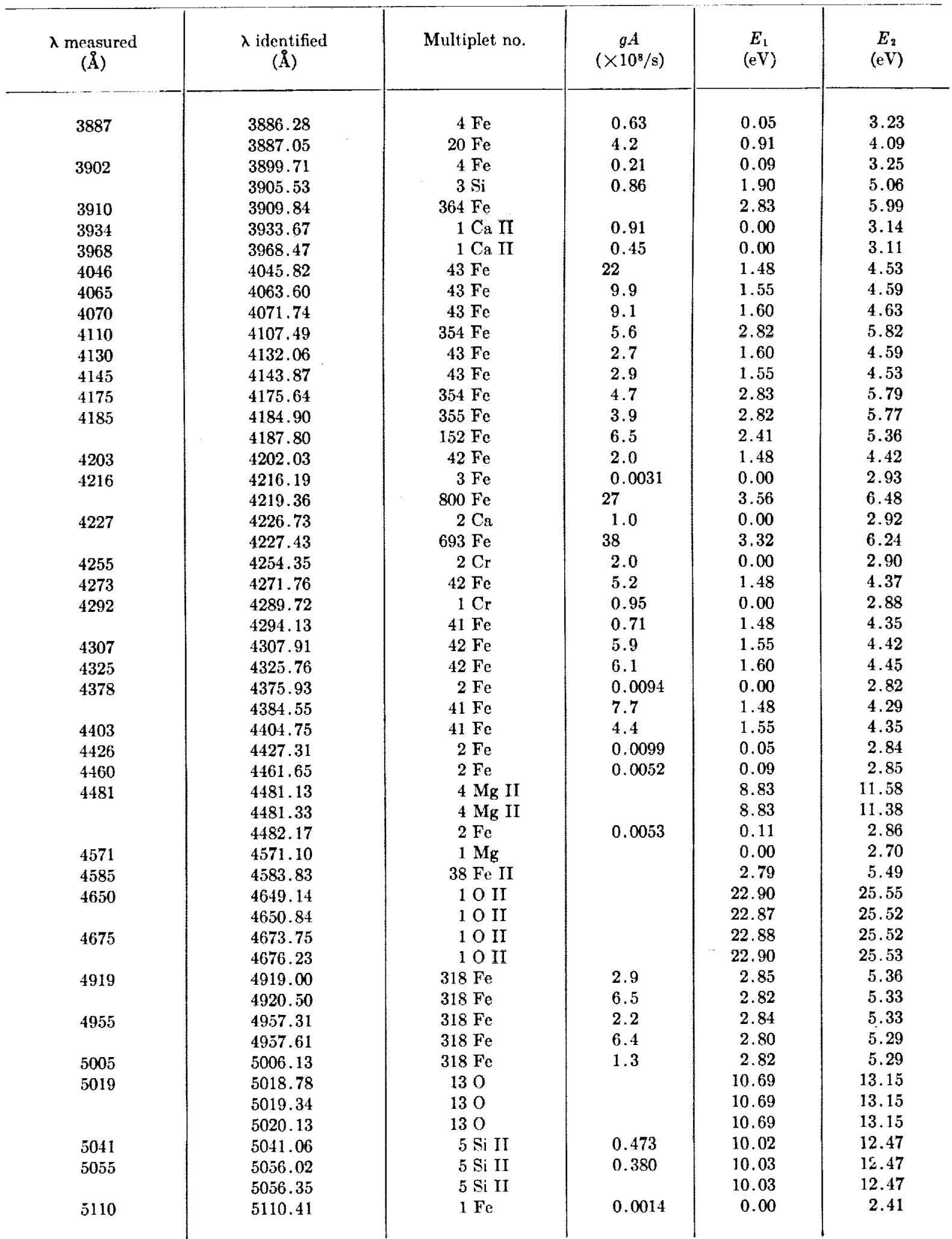


TABLE 4.-Wavelength Identifications of Perseid Meteor-Continued

\begin{tabular}{|c|c|c|c|c|c|}
\hline $\begin{array}{c}\lambda \text { measured } \\
(\AA)\end{array}$ & $\begin{array}{c}\lambda \text { identified } \\
(\AA)\end{array}$ & Multiplet no. & $\left(\begin{array}{c}g A \\
\left(\times 10^{8} / \mathrm{s}\right)\end{array}\right.$ & $\begin{array}{c}E_{1} \\
(\mathrm{cV})\end{array}$ & $\begin{array}{c}E_{2} \\
(\mathrm{cV})\end{array}$ \\
\hline \multirow{3}{*}{5142} & 5139.26 & $383 \mathrm{Fe}$ & 1.6 & 2.99 & 5.39 \\
\hline & 5139.48 & $383 \mathrm{Fc}$ & 2.0 & 2.93 & 5.33 \\
\hline & 5142.93 & $16 \mathrm{Fe}$ & & 0.95 & 3.35 \\
\hline \multirow[t]{6}{*}{5170} & 5166.29 & $1 \mathrm{Fe}$ & & 0.00 & 2.41 \\
\hline & 5167.34 & $2 \mathrm{Mg}$ & 1.2 & 2.70 & 5.09 \\
\hline & 5167.49 & $37 \mathrm{Fe}$ & 0.26 & 1.48 & 3.87 \\
\hline & 5168.90 & $1 \mathrm{Fe}$ & & 0.05 & 2.44 \\
\hline & 5171.60 & $36 \mathrm{Fe}$ & 0.12 & 1.48 & 3.86 \\
\hline & 5172.70 & $2 \mathrm{Mg}$ & 3.5 & 2.70 & 5.09 \\
\hline 5184 & 5183.62 & $2 \mathrm{Mg}$ & 6.4 & 2.70 & 5.09 \\
\hline \multirow[t]{2}{*}{5226} & 5226.88 & $383 \mathrm{Fe}$ & 2.0 & 3.03 & 5.39 \\
\hline & 5227.19 & $37 \mathrm{Fe}$ & 0.27 & 1.55 & 3.91 \\
\hline \multirow[t]{2}{*}{5269} & 5269.54 & $15 \mathrm{Fe}$ & 0.098 & 0.86 & 3.20 \\
\hline & 5270.36 & $37 \mathrm{Fe}$ & 0.20 & 1.60 & 3.94 \\
\hline \multirow[t]{5}{*}{5328} & 5328.05 & $15 \mathrm{Fe}$ & 0.087 & 0.91 & 3.23 \\
\hline & 5328.53 & $37 \mathrm{Fe}$ & 0.052 & 1.55 & 3.87 \\
\hline & 5328.98 & $12 \mathrm{O}$ & & 10.69 & 13.01 \\
\hline & 5329.59 & 120 & & 10.69 & 13.01 \\
\hline & 5330.66 & $12 \mathrm{O}$ & & 10.69 & 13.01 \\
\hline 5343 & 5341.03 & $37 \mathrm{Fe}$ & 0.042 & 1.60 & 3.91 \\
\hline 5370 & 5371.49 & $15 \mathrm{Fe}$ & 0.062 & 0.95 & 3.25 \\
\hline 5428 & 5429.70 & $15 \mathrm{Fe}$ & 0.039 & 0.95 & 3.23 \\
\hline 5453 & 5455.61 & $15 \mathrm{Fe}$ & 0.022 & 1.01 & 3.27 \\
\hline 5525 & 5528.46 & $9 \mathrm{Mg}$ & 1.6 & 4.33 & 6.56 \\
\hline \multirow[t]{2}{*}{5570} & 5569.62 & $686 \mathrm{Fc}$ & 2.4 & 3.40 & 5.62 \\
\hline & 5572.85 & $686 \mathrm{Fe}$ & 3.4 & 3.38 & 5.60 \\
\hline \multirow[t]{2}{*}{5586} & 5586.76 & $686 \mathrm{Fe}$ & 4.2 & 3.35 & 5.56 \\
\hline & 5588.75 & $21 \mathrm{Ca}$ & 5.4 & 2.51 & 4.72 \\
\hline 5775 & 5772.26 & $17 \mathrm{Si}$ & & 5.06 & 7.20 \\
\hline 5800 & 5797.91 & $9 \mathrm{Si}$ & & 4.93 & 7.09 \\
\hline \multirow[t]{2}{*}{5893} & 5889.95 & $1 \mathrm{Na}$ & 1.8 & 0.00 & 2.10 \\
\hline & 5895.92 & $1 \mathrm{Na}$ & 0.90 & 0.00 & 2.09 \\
\hline 5960 & 5957.61 & $4 \mathrm{Si} \mathrm{II}$ & 0.356 & 10.02 & 12.09 \\
\hline 5980 & 5978.97 & $4 \mathrm{Si}$ II & 0.527 & 10.03 & 12.09 \\
\hline 6000 & 5999.47 & $16 \mathrm{~N}$ & & 11.55 & 13.61 \\
\hline 6123 & 6122.22 & $3 \mathrm{Ca}$ & 1.2 & 1.88 & 3.89 \\
\hline \multirow[t]{4}{*}{6155} & 6154.23 & $5 \mathrm{Na}$ & & 2.09 & 4.70 \\
\hline & 6155.99 & 100 & & 10.69 & 12.70 \\
\hline & 6156.78 & 100 & & 10.69 & 12.70 \\
\hline & 6158.20 & 100 & & 10.69 & 12.70 \\
\hline 6348 & 6347.09 & $2 \mathrm{Si} \mathrm{II}$ & 0.31 & 8.09 & 10.03 \\
\hline 6371 & 6371.36 & $2 \mathrm{Si} \mathrm{II}$ & 0.387 & 8.09 & 10.02 \\
\hline 6440 & 6439 & $18 \mathrm{Ca}$ & 3.2 & 2.51 & 4.43 \\
\hline \multirow[t]{3}{*}{6456} & 6453.64 & 90 & & 10.69 & 12.61 \\
\hline & 6454.48 & 90 & & 10.69 & $12.6 \mathrm{I}$ \\
\hline & 6456.01 & 90 & & 10.69 & 12.61 \\
\hline \multirow[t]{4}{*}{6483} & 6481.73 & $21 \mathrm{~N}$ & 0.0662 & 11.70 & 13.61 \\
\hline & 6482.74 & $21 \mathrm{~N}$ & & 11.70 & 13.61 \\
\hline & 6483.75 & $21 \mathrm{~N}$ & & 11.70 & 13.61 \\
\hline & 6484.88 & $21 \mathrm{~N}$ & 0.2725 & 11.70 & 13.61 \\
\hline 6562 & 6562.82 & $1 \mathrm{H}$ & & 10.15 & 12.04 \\
\hline
\end{tabular}




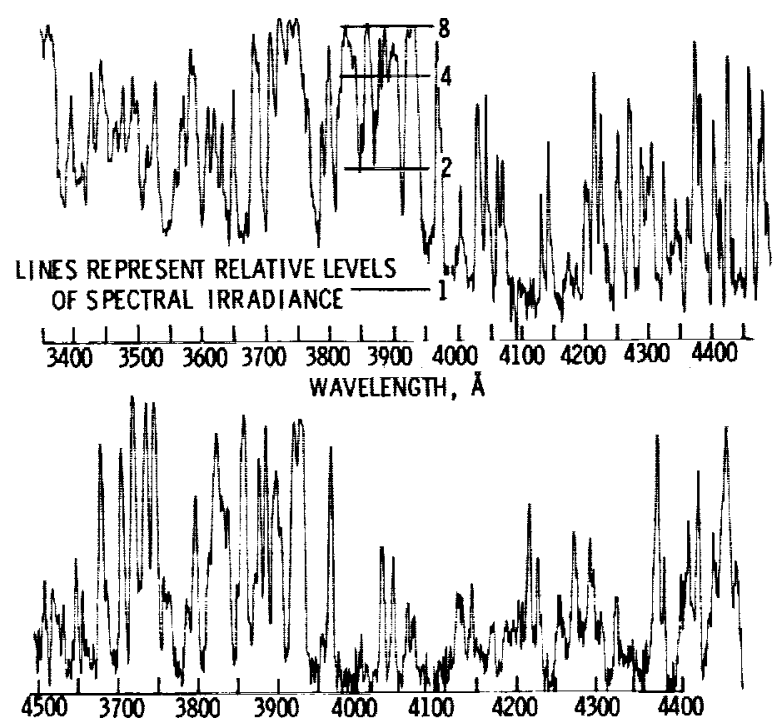

Figure 5. - Spectral irradiance from sporadic meteor.

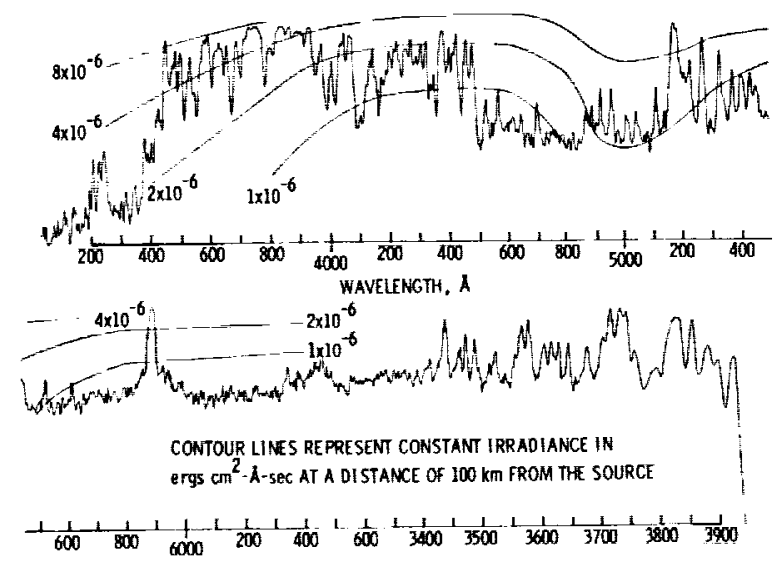

Figure 6.-Spectral irradiance from Taurid meteor.

several different energy levels is taken to be indicative that the excited state populations follow a Boltzmann distribution. In particular, the relative populations of the neutral excited states in the encrgy range of 2 to $6 \mathrm{eV}$ are obtained. This is the energy region in which the number of lines in a good metcor spectrum allows one to measure the populations. Iron, because of the large number of lines of different cnergy levels, is a convenient reference element for these measurements.

As has been shown by Ceplecha $(1964,1967)$, Harvey (1970) and Millman (1932, 1935), the

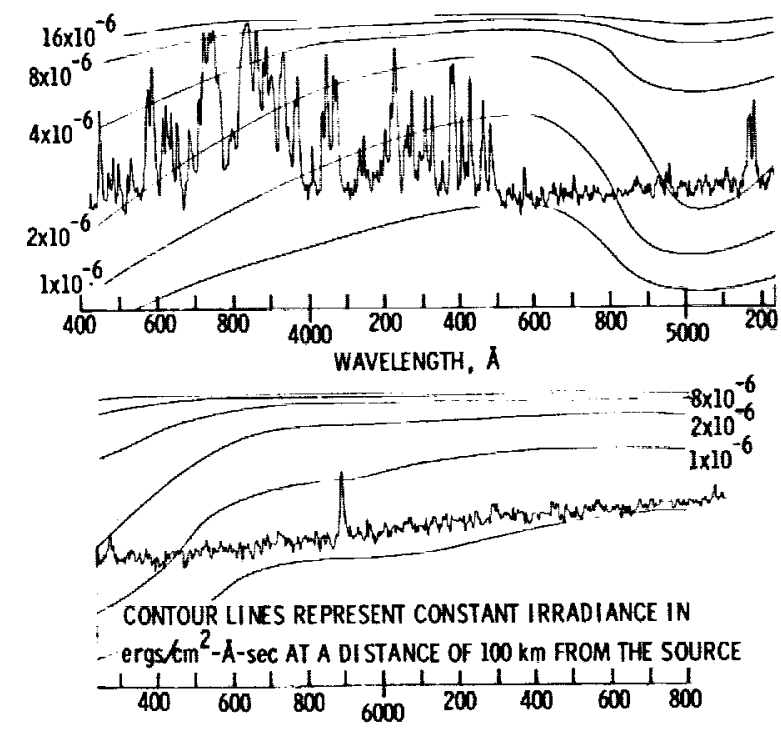

Figure 7.-Spectral irradiance from Geminid meteor.

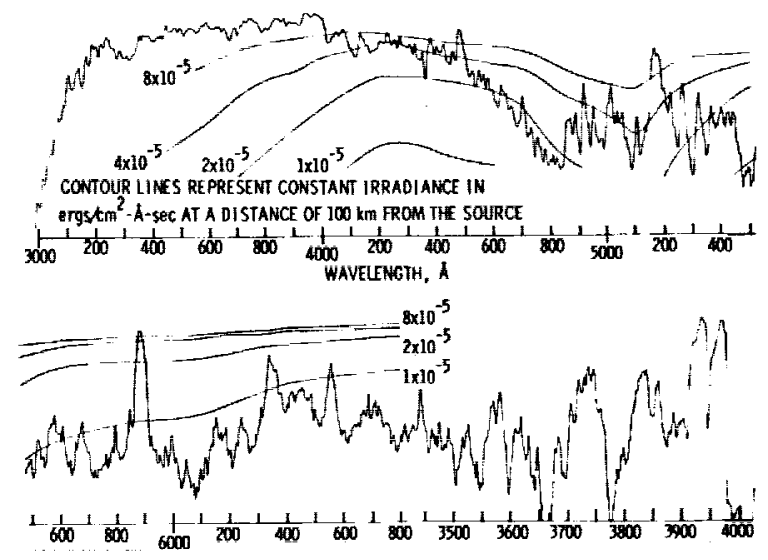

Figure 8.- Spectral irradiance from Perseid meteor.

population distribution in this range is similar to that of a Boltzmann distribution. Espccially for the fainter metcors, we can assume that all of the states are underpopulated relative to a gas in local thermodynamic equilibrium since the observed states are depopulated by radiative transfers (which occur quickly, compared to time between collisions). We can also assume that the very high $(>6 \mathrm{eV})$ encrgy states are overpopulated relative to the lower energy states in local thermodynamic cquilibrium (LTE) because of the high initial relative velocities of the ablated meteor atoms. However, as the underpopulation 
due to radiative transfer affects all the atoms and little radiation is observed from very high energy states (excluding ionic radiation), we can ignore these effects if we restrict ourselves to working on a relative basis and in a limited energy range.

The analysis, then, simply consists of selecting pairs of lines within the 2 to $6 \mathrm{cV}$ energy range and computing an "effective meteor radiation temperature" $T^{*}$. Thus, $T^{*}$ is calculated from

$$
\frac{I_{i n}}{I_{j l}}=\frac{h \nu_{i n} \Lambda_{i} A_{i n}}{h \nu_{j l} N_{j} A_{j l}}=\frac{h \nu_{i n} g_{i} A_{i n}}{h \nu_{j l} l g_{j} A_{j l}} e^{-\left(E_{i}-E_{i}\right) / k T *}
$$

where $I_{i n}$ is the intensity of radiation for the transition from the $i$ th to the $n$th atomic state, $I_{j l}$ is the intensity of radiation for the transition from the $j$ th to the 7 th atomic state, $N_{i}$ is the number of particles in the ith atomic state with energy $E_{i}$ above the ground state, $h$ is Planck's constant, $\nu_{i n}$ is the frequency of observed radiation for the transition from the $i$ th to the $n$th state, $g_{i}$ is the statistical weight of the $i$ th state, $A_{\text {in }}$ is the Einstein probability coefficient for the transition from the $i$ th to the $n$th state, and $k$ is the Boltzmann constant. The results of the calculations are shown in tables 5 to 8 for the sporadic, the Taurid, the Geminid, and the Perseid meteors, respectively. Excitation energies comparable to temperatures of hundreds of thousands of degrees are arailable in the initial meteor atomic collisions. General agreement in the range of $T^{*}$ calculated from different pairs of lines indieates that the first atomic collisions do not dominate the meteor radiation process.

In general, table 5 reflects the advantage of high spectral resolution and its resultant effect

TABle 5.-Sporadic "Effective Meteor Radiation Temperature"

\begin{tabular}{ccc}
\hline Element & $\begin{array}{c}\text { Lines } \\
(\AA)\end{array}$ & $\begin{array}{c}\text { Temperature } \\
\left({ }^{\circ} \mathrm{K}\right)\end{array}$ \\
\cline { 1 - 2 } Fe I & 4384,4376 & 2280 \\
Fe I & 3570,3491 & 2453 \\
Fe I & 3648,3680 & 2880 \\
Fe I & 4144,4216 & 2540 \\
Fe I & 4404,4427 & 2565 \\
Fe I & ar & $2544 \pm 197$ \\
& & standard error \\
\hline
\end{tabular}

TABle 6.-Taurid "Effective Meleor Radiation Temperature"

\begin{tabular}{lcc}
\hline Element & $\begin{array}{c}\text { Lines } \\
(\AA)\end{array}$ & $\begin{array}{c}\text { Temperature } \\
\left({ }^{\circ} \mathrm{K}\right)\end{array}$ \\
\hline Fe I & 4384,4376 & 2490 \\
Fe I & 3570,3441 & 3880 \\
Fe I & 3648,3680 & 3360 \\
Fe I & 4405,4427 & 2740 \\
Fe I & 4046,4427 & 2570 \\
Fe I & 3631,3707 & 3640 \\
Fe I & 3758,3719 & 3870 \\
Fe I & 3581.3719 & 3950 \\
Fe I & 4216,4046 & 2260 \\
& av & $3196 \pm 640$ \\
& & standard error \\
\hline
\end{tabular}

TABLe 7.-Geminid "Effective Veteor Radiation Temperalure"

\begin{tabular}{ccc} 
Element & $\begin{array}{c}\text { Lines } \\
(\AA)\end{array}$ & $\begin{array}{c}\text { Temperature } \\
\left({ }^{\circ} \mathrm{K}\right)\end{array}$ \\
\hline Fe I & 4384,4376 & 2560 \\
Fe I & 3648,3680 & 3680 \\
Fe I & 4405,4427 & 2745 \\
Fe I & 4046,4427 & 2800 \\
Fe I & 3631,3707 & 4680 \\
Fe I & 3581,3719 & 4150 \\
Fe I & 4046,4216 & 2500 \\
& ar & $3302 \pm 776$ \\
& & standard error \\
\hline
\end{tabular}

Table 8.-Perseid "Effective Meteor Radiation Temperature"

\begin{tabular}{lcc}
\hline Element & $\begin{array}{c}\text { Lines } \\
(\AA)\end{array}$ & $\begin{array}{c}\text { Temperature } \\
\left({ }^{\circ} \mathrm{K}\right)\end{array}$ \\
\hline Fe I & 4384,4376 & 2780 \\
Fe I & 4405,4427 & 2790 \\
Fe I & 4046,4427 & 2670 \\
Fe I & 4046,4216 & 2500 \\
Fe I & 5139,5110 & 3970 \\
& ar & $2942 \pm 525$ \\
Fa I & 5153,5893 & 10,770 \\
Ca I & 6439,4226 & 4470 \\
Mg I & 5528,5184 & 12,810 \\
\hline
\end{tabular}


on "effective radiation temperature" measurements. The Taurid mensurements are compromised somewhat by overexposure. The Geminid measurements are relatively clean, while the Perseid measurements are poor because of bad imagery.

The abundanees of elements relative to iron were computed by using the "effective radiation temperatures." That is, the atomic ratio of element $a$ to element $b$, where element " $b$ " is always iron, was obtained from

$$
\frac{N_{a}}{N_{b}}=\frac{I_{i n a}}{I_{j l b}} \frac{B(T)_{a}}{B(T)_{b}} \frac{g_{j b} A_{j l b} \nu_{j l b}}{g_{i a} A_{i n a} \nu_{i n a}} e^{-\left(E_{j b}-E_{i a}\right) / k T^{*}}
$$

where $Y_{a}$ is the number of atoms of element $a$, $X_{b}$ is the number of atoms of iron, $B(T)_{a}$ is the partition function of element $a$, and $B(T)_{b}$ is the partition function of iron. Partition functions from Corliss (1962) were used. Iron was used as the reference element its prevalence in neutral spectra, and because of the large number of lines suitable for spectral measurements.

The ratios of the poak intensities of the relevant lines were used. The radiation of the element in question was compared to that of an iron line in the same spectral region, of similar energy level, and of comparable line strength where possible. The silieon determinations are highly unecrtain berause of the weakness of the $3905 \mathrm{~A}$ line and the blending of other lines in this region of the spectrum. The element ratios calculated from the spectral measurements of the four meteors are presented in tables 9 to 12. "Derived compositions" bised on the element ratios and typical metcorite oxygen abundances are also listed in the tables.

A de are was used as an empirical tool for data imalysis. Some diffieulty has been experieneed in obtaining are temperatures as low as some of the "equivalent radiation temperatures" of the meteors. Nerertheless, there is good generil qualitative angerment between a de are and neutral moteor spectrit. The are studies indieate thit the individual "equivilent radiation temperatures" obtained by this type of mothod are uneertain to sereral hundred degrees Folvin for farorible line intensity measurements. The studies also show that reisonable silieon abundaner moisurements are pessible with the 390 i in line with high resolution and good plate quality,
TABLE 9.-Element Ratios and "Derived Composition" of Sporadic Meteor

\begin{tabular}{c|c}
\hline $\begin{array}{c}\text { Element ratios } \\
\text { (atomic) }\end{array}$ & $\begin{array}{c}\text { Derived composition } \\
\text { (percent by weight) }\end{array}$ \\
\hline$\frac{\mathrm{Ni}}{\mathrm{Fe}}=0.15$ & $\mathrm{Fe}-68$ \\
$\mathrm{Ni}-10$ \\
$\mathrm{Ca}-0.0058$ \\
$\mathrm{Mn}-0.13$ \\
$\mathrm{Cr}-0.015$ \\
$\mathrm{Mg}-0.19$ \\
$\mathrm{Fi}-9.8$ \\
$\mathrm{O}-11$ \\
$\frac{\mathrm{Mn}}{\mathrm{Fe}}=1.8 \times 10^{-3}$ \\
$\frac{\mathrm{Cr}}{\mathrm{Fe}}=2.2 \times 10^{-4}$ \\
$\frac{\mathrm{Mg}}{\mathrm{Fe}}=1.16 \times 10^{-3}$
\end{tabular}

\section{RADIATION}

Four meteor spectra, each of more than 100 lines, have been reduced and analyzed. These four spectra of a sporadic, a Taurid, a Geminid, and a Perseid metcor may be taken as generally representative of bright metcor spectra in the optical range. The analyses of these spectra have shown a surprising simplicity and consistency of the radiation process for the neutral radiation.

The populations of the neutral exeited states in the 2 to $6 \mathrm{eV}$ encrgy range have been found to be consistent with that of a Boltzmann distribution within the experimental error. This may be taken as an indication that this type of radiation is produeed by a gas that is near equilibrium. A physical conecpt that can be correlated with these moisured results is as follows: meteoric gas is initially encrgized by the passage and ablation of a metcoroid. The following "relaxation and mixing" of this morgized gas probably involves hundreds of collisions within milliscconds. The relaxition and mixing can be considered in terms of the downward relocity cascade of meteoric atoms (and impacted atmospheric molecules) through a scries of collisions with atmospheric molecules. For a simple elastic hard sphere 
TABle 10.-Element Ratios and "Derived Composition" of Taurid Meteor

\begin{tabular}{|c|c|}
\hline $\begin{array}{l}\text { Element ratios } \\
\text { (atomic) }\end{array}$ & $\begin{array}{l}\text { Derived composition } \\
\text { (percent by weight) }\end{array}$ \\
\hline $\begin{array}{l}\frac{\mathrm{Ni}}{\mathrm{Fe}}=0.106 \\
\frac{\mathrm{Ca}}{\mathrm{Fe}}=1.5 \times 10^{-4} \\
\frac{\mathrm{Mn}}{\mathrm{Fe}}=3.2 \times 10^{-4} \\
\frac{\mathrm{Cr}}{\mathrm{Fe}}=5.1 \times 10^{-4} \\
\frac{\mathrm{Mg}}{\mathrm{Fe}}=1.8 \\
\frac{\mathrm{Si}}{\mathrm{Fe}}=4.6 \\
\frac{\mathrm{Na}}{\mathrm{Fe}}=8.1 \times 10^{-5} \\
\frac{\mathrm{Al}}{\mathrm{Fe}}=1.7 \times 10^{-3}\end{array}$ & $\begin{array}{c}\mathrm{Fe}-13 \\
\mathrm{Ni}-1.6 \\
\mathrm{Ca}-0.0015 \\
\mathrm{Mn}-0.0043 \\
\mathrm{Cr}-0.0068 \\
\mathrm{Mg}-10 \\
\mathrm{Si}-30 \\
\mathrm{Na}-0.00045 \\
\mathrm{Al}-0.11 \\
\mathrm{O}-44\end{array}$ \\
\hline
\end{tabular}

model, the most probable energy transfer from an incident atom to a target atom has been calculated to be of the order of 25 percent of the incident atom energy. For a simple scrics of collisions with atmospheric molecules at rest, more than 20 collisions are required for a typical meteoric atom of $30 \mathrm{~km} / \mathrm{s}$ initial velocity to "cool" to a velocity of $1.26 \mathrm{~km} / \mathrm{s}$ (which corresponds to a $4000^{\circ} \mathrm{K}$ gas velocity). This simple cascade is a minimum case, as both a more realistic atomic scattering potential and collisionincreased atmospheric-molecule velocity will decrease the most probable energy transfer to less than 25 percent of the incident atom energy. However, even the minimum number of collisions is sufficient for the velocity distribution to approach that of an equilibrium gas. In some cases, a Boltzmann distribution has been shown to be closely approached after only three collisions per particle (Kittel, 1958).

Some complication is introduced because the radiation that is photographed with a streak camera or spectrograph is a composite of the
TABLe 11.-Element Ratios and "Derived Composition" of Geminid Meteor

\begin{tabular}{l|c}
$\begin{array}{c}\text { Element ratios } \\
\text { (atomic) }\end{array}$ & $\begin{array}{c}\text { Derived composition } \\
\text { (percent by weight) }\end{array}$ \\
\hline$\frac{\mathrm{Ni}}{\mathrm{Fe}}=0.114$ & $\mathrm{Fe}-13$ \\
$\mathrm{Ni}-1.7$ \\
$\mathrm{Ca}-0.02$ \\
$\mathrm{Fe}=2.0 \times 10^{-3}$ & $\mathrm{Cr}-0.0068$ \\
$\mathrm{Mg}-12$ \\
$\mathrm{Mn}$
\end{tabular}

radiation from the series of collisions. That is, this radiation results initially from a few very high energy collisions, from many more collisions of moderate energy at a longer and later instant when the gas is approaching equilibrium, of many, many more collisions of moderately low energy at a still later time when the gas is very close to an equilibrium velocity distribution, and, finally, of still more collisions when the gas is practically in equilibrium, but still cooling and radiating as the meteor wake. This interpretation is in general agreement with radiation times and energy levels observed in wake and flare radiation (Harvey, 1971b).

It appears, from the time-integrated spectral measurements, that superposition of radiation from all of the radiation times results in a spectrum that is similar to that of a gas near equilibrium. This probably results from the initial collisions being too few and having too broad a spectrum (many energy levels can be excited) to dominate the radiation, and the later wake radiation being too energy-limited to dominate 
TABLE 12.-Element Ratios and "Derived Composition" of Perseid Meteor

\begin{tabular}{c|c}
\hline $\begin{array}{c}\text { Element ratios } \\
\text { (atomic) }\end{array}$ & $\begin{array}{c}\text { Derived composition } \\
\text { (percent by weight) }\end{array}$ \\
\hline$\frac{\mathrm{Ca}}{\mathrm{Fe}}=3.9 \times 10^{-4}$ & $\mathrm{Fe}-13$ \\
$\frac{\mathrm{Mn}}{\mathrm{Fe}}=4.9 \times 10^{-4}$ & $\mathrm{Mn}-0.0037$ \\
$\mathrm{Cr}-0.0064$ \\
$\mathrm{Mg}-3.9$ \\
$\frac{\mathrm{Cr}}{\mathrm{Fe}}=1.1 \times 10^{-3}$ & $\mathrm{Si}-36$ \\
$\mathrm{Mg}-0.00028$ \\
$\frac{\mathrm{Fg}}{\mathrm{Fe}}=0.68$ & $\mathrm{H}-$ ? \\
$\frac{\mathrm{Si}}{\mathrm{Fe}}=5.6$ & \\
$\frac{\mathrm{Na}}{\mathrm{Fe}}=5.2 \times 10^{-5}$ & \\
\hline
\end{tabular}

the spectrum. Thus, an intermediate case must dominate. The relatively few initial high energy collisions will tend to average the many inefficient low energy collisions to an intermediate case.

However, this does not mean that all meteor radiation is simple or straightforward. Resonance or nonequilibrium effects are observed in all detailed studies of radiation sources, be they flames, ares, stellar atmospheres, or other sources. This obviously is the case with the strong ionic emissions in bright, fast meteors. This is cvideneed by the absence of such radiation in faint meteors, and the absence of normally expected ionic lines. Several articles concerning $H$ and $K$ radiation are in the literature (Harvey, 1971b; Hoffman, 1971; Hoffman and Longmire, 1968; Rajehl, 1963). The obscrved Mg II and Si II radiation is even more anomalous because of its higher energy levels and the absenee of many normally strong ion lines.

As significant as the general agrement in "effective radiation temperatures," shown in tables 5 to 8 , is the fact that there is no identificd neutral iron radiation that is not in gencral agreement with a Boltzmann distribution. The same is true of other neutral spectra where there are enough suitable lines to make a meaningful measurement. Thus, all of the neutral line radia- tion appears well behaved, the expected lines are present and are at the expected intensity, unexpeeted lines are not present.

We may conelude, then, that optical meteor radiation does not result from just the initial collisions of meteoric atoms with atmospheric molecules, but by a complete energy-cascadevia-collisions process. Since this involves at least tens to hundreds of collisions, it is basically a statistical process, and can be treated in terms of equilibrium distributions (over limited ranges) and deviations from equilibrium. The radiation can also be treated in terms of individual atomic cross sections but this requires even more detailed knowledge of the metcor conditions.

\section{COMPOSITION}

The measurement of composition need not be strongly dependent upon the assumption that the excited state populations are given by a Boltzmann distribution. The composition measurements require only that the meteor radiation processes of the relevant spectral lines be basically the same. That is, the population distribution of excited states of different atoms need to be similar. The distribution does not even need to be known very well, if the encrgy levels of the excited states chosen for measurement are close together.

If the metcoric excitation process is selective with respect to one atom over another, then problems may arise. However, since optical meteor excitation is basically collisional, that is, no radiative coupling occurs, it is unlikely that neutral radiation excitation is significantly selective in light of the consistency of the "effective radiation temperature measurements." Sclcetivity does, of course, show up in the ionic radiation and renders these lines unstable for abundance measurements. This need not prevent the use of neutral radiation for composition measurements.

Perhaps the most notable result of the mrasurement of the four meteors is that they are indicative of nickel-iron or stony metcorite composition. However, it should be noted that the sodium and calcium abundances are very low compared to cosmic or motcoritc abundances. No effects such as neutral atom depletion by ionization, incomplete dissociation, or self absorp- 
tion in the metcor plasma have been considered in these preliminary abundance values. Such effects will be considered in a subsequent paper.

The significant elements which have not been obtained from the analysis are oxygen, carbon, and hydrogen (silicon has already been discussed). Reasonable values for the abundances of oxygen can be obtained from those elements with which it is combined in meteorites. Carbon is not expected in major abundances, but is important for studies of meteorite origin and evolution. The best hope for measured carbon abundances is probably the very difficult one of measuring carbon band systems. Needless to say, observational difficulties for these measurements are extreme. A somewhat unexpected result is the strength of $\mathrm{H} \alpha$ in bright, fast meteor spectra. Little hydrogen would generally be expected after repeated passes of a meteoroid at distances of less than $1 \mathrm{AU}$ from the Sun. Both the high energy level of the excited state and the low atomic weight (and hence low kinetic energy) are significant aspects of the hydrogen radiation which will require serious consideration.

\section{CONCLUDING REMARKS}

Four spectra, which are believed to be generally representative of the spectra of brighter meteors obtained by the Faint Meteor Spectra Patrol, have been reduced and analyzed. The neutral line radiation from these meteors was determined to be similar to that of a gas in equilibrium at a relatively low temperature. These results demonstrate that the powerful concept of local thermodynamic equilibrium can be fruitfully applied to meteor spectroscopy. In the past, poor quality of spectral data and the dominance of anomalous ionic radiation in much of the better spectra have served as deterrents to detailed quantitative analysis of meteor spectra. Thus, it is hoped that the present results will hasten the transition of meteor spectroscopy from primarily qualitative studies which have characterized it in the past to detailed quantitative studies which improved data now warrant.

The "derived composition" of the slow sporadic meteor appears to be generally similar to that of a typical nickel-iron meteorite. The "derived compositions" of the three shower meteors appear to be similar to and seem to indicate stony meteorite composition. These "derived compositions," although presently the most comprehensive direct data on meteor composition, are early results that are expected to be rapidly supplemented by additional data of even greater quality and quantity.

\section{REFERENCES}

Ayers, W. G., McCrosky, R. E., and Shao, C. -Y., 1970. Photographic observations of 10 artificial meteors, Smithson. Astrophys. Obs. Spec. Rept. No. 317, 1-40.

Ceplechs, Z., 1964. Study of a bright meteor flare by means of emission curve of growth, Bull. Astron. Inst. Czech., 15, 102-112.

—, 1965. Complete data on bright metcor 32281, Bull. Astron. Inst. Czech., 16, 88-101.

- , 1967. Spectroscopic analysis of iron meteoroid radiation, Bull. Astron. Inst. Czech., 18, 303-310.

Cook, A. F., JACChiA, L. G., AND MCCrosky, R. E., 1963. Luminous efficiency of iron and stone asteroidal meteors, Smithson. Contrib. Astrophys., 7, 209-220.

Conliss, C. H., 1962. Ionization in the plasma of a copper arc, J. Res. Nat. Bur. Standards, 66A, $169-175$.

Conliss, C. H., and Bozman, W. R., 1962. Experimental transition probabilities for spectral lines of seventy elements, NBS Monograph 53, U.S. Dept. of Commerce, Washington.

D'Aiutolo, C. T., Kinard, W. H., and Nauman, R. J., 1967. Recent NASA meteoroid penetration results from satellites, Smithson. Conirib. Astrophys., 11, 239-251.

Fiterman, L., 1964. Atmospheric attenuation model, 1964, in the ultraviolet, visible, and infrared regions for altitudes to $50 \mathrm{~km}, U . S$. Air Force Environ. Res. Papers No. 46 (AFCRL64-740), Sept. 1964, 1-40.

Griem, H. R., 1964. Plasma Spectroscopy, McGraw-Hill Book Co., New York, 580 pp. 
Grygar, J., Kohoutek, I., and Plavcovi, Z., 1968. Simultaneous radar and optical observation of meteors at Ondrejov in 1962, in Physics and Dynamics of Meteors, edited by $\check{L}$. Kresík and P. M. Millman, D. Reidel Publ. Co., Dordrecht, Holland, 63-69.

Haldiday, I, 1961. A study of spectral line identifieations in Perseid meteor spectra, Publ. Dominion Obs., 25, 1-16.

- 1969. A study of ultraviolet meteor spectra, Publ. Dominion Obs., 25, 315-322.

Hanvey, G. A., 1967a. Photometry of spectrograms of three artificial meteors, NASA Tech. Note D-3930, 1-30.

- 1967b. A method of slitless absolute spectral photometry, NASA Tech. Note D-3765, 1-20.

- 1970 . Spectra of faint optical meteors, unpublished.

- 1971a. The NASA LRC faint meteor spectra patrol, NASA Tech. Note D-6298, 1-25.

, $1971 \mathrm{~b}$. The calcium $H$ - and $K$-line anomaly in meteor spectra, Astrophys. $J$., 165, 669-671.

Hoffuna, H. S., 1971. Ionic spectra of meteors, Astrophys. J., 163, 393-403.

Hoffman, H. S., ANd Longmire, M. S., 1968. Meteor ion spectra, Nature, 218, 858-859.

Jacchia, I. G., Verniani, F., and Briggs, F., 1967. An analysis of the atmospheric trajectories of 413 precisely reduced photographic meteors, Smithson. Contrib. Astrophys., 10, 1-139.

KitTel, C., 1958. Elementary Slalistical Physics, J. Wiley and Sons, New York, 228 pp.

LindBlad, B. A., 1963. The relation between visual magnitudes and the durations of radar echoes, Smilhson. Contrib. Astrophys., 7, 27-39.

Mrllman, P. M., 1932. An analysis of meteor spectra, Harvard College Obs. Ann., 82, 113-146.

- 1935. An analysis of meteor spectra: second paper, Harvard College Obs. Ann., 82, 149-177.

- 1967. Some characteristics of the major meteor showers, Smithson. Contrib. Astrophys., 11, 105-108.

Mоове, C. E., 1945. A multiplet table of astrophysical interest, Conirib. Princeton Univ. Obs, No. 20.

RAJChl, J., 1963. A short note on meteor spectra with low dispersion, Smithson. Contrib. Astrophys., 7, 155-156. 
